\title{
Seasonal Synchronization of a Simple Stochastic Dynamical Model Capturing EI Niño Diversity
}

\author{
Sulian Thual, Andrew Majda, AND NAn Chen \\ Department of Mathematics, and Center for Atmosphere Ocean Science, Courant Institute of Mathematical Sciences, \\ New York University, New York, New York
}

(Manuscript received 16 March 2017, in final form 3 September 2017)

\begin{abstract}
Recently, a simple stochastic dynamical model was developed that automatically captures the diversity and intermittency of El Niño-Southern Oscillation (ENSO) in nature, where state-dependent stochastic wind bursts and nonlinear advection of sea surface temperature (SST) are coupled to simple oceanatmosphere processes that are otherwise deterministic, linear, and stable. In the present article, it is further shown that the model can reproduce qualitatively the ENSO synchronization (or phase locking) to the seasonal cycle in nature. This goal is achieved by incorporating a cloud radiative feedback that is derived naturally from the model's atmosphere dynamics with no ad hoc assumptions and accounts in simple fashion for the marked seasonal variations of convective activity and cloud cover in the eastern Pacific. In particular, the weak convective response to SSTs in boreal fall favors the eastern Pacific warming that triggers El Niño events while the increased convective activity and cloud cover during the following spring contributes to the shutdown of those events by blocking incoming shortwave solar radiations. In addition to simulating the ENSO diversity with realistic non-Gaussian statistics in different Niño regions, the eastern Pacific moderate and super El Niño and the central Pacific El Niño and La Niña show a realistic chronology with a tendency to peak in boreal winter as well as decreased predictability in spring consistent with the persistence barrier in nature. The incorporation of other possible seasonal feedbacks in the model is also documented for completeness.
\end{abstract}

\section{Introduction}

El Niño-Southern Oscillation (ENSO) is the largest global climate signal on interannual time scales, with dramatic worldwide ecological and social impacts. It consists of alternating periods of anomalously warm El Niño conditions and cold La Niña conditions every 2$7 \mathrm{yr}$, with considerable irregularity in amplitude, duration, temporal evolution, and spatial structure of these events. Its dynamics in the equatorial Pacific result largely from coupled interactions between the ocean and atmosphere at an interannual time scale and planetary scale (Neelin et al. 1998; Clarke 2008).

One of the most remarkable yet elusive characteristics of ENSO is its partial synchronization to the seasonal cycle, with a tendency for El Niño events to develop during the boreal spring to fall season, peak in boreal winter, and shut down the following spring. A brief introductory illustration of this seasonal synchronization

Corresponding author: Sulian Thual, sulian.thual@gmail.com in observations is provided hereafter in section 2a. Associated sea surface temperature (SST) anomalies, for example, reach their maximum in the central to eastern Pacific within the months of November-January for most recorded events. This synchronization is a clear indication that the seasonal cycle in the equatorial Pacific Ocean and atmosphere plays a major role in ENSO's dynamics. Understanding this seasonal synchronization is also essential for the prediction of $\mathrm{El}$ Niño and its possible worldwide teleconnections. For instance, many ENSO forecast schemes show a marked decay in skill in boreal spring (the so-called spring barrier) paralleled by a reduction in the persistence of observed equatorial Pacific SST anomalies (Torrence and Webster 1998; Levine and McPhaden 2015). The causes and nature of this seasonal skill dependence still remain as an open question.

The exact mechanisms that cause the seasonal synchronization of ENSO are not yet fully understood, though they have been studied in a number of publications. The intimate dependency of ENSO on the 
seasonal cycle presents a major challenge to state-of theart coupled general circulation models (CGCMs). For instance, most of those CGCMs still show deficiencies in simulating the ENSO amplitude and frequency, spatial structure, and seasonal synchronization due to systematic biases in the mean climate and seasonal cycle of the tropical Pacific (Guilyardi 2006; Lloyd et al. 2012; Bellenger et al. 2014). Meanwhile, the seasonal synchronization of ENSO is captured in several simpler models based on different recipes. In those simple models the seasonal cycle is usually prescribed and modulates the stability of the equatorial Pacific oceanatmosphere system, resulting in ENSO phase locking (i.e., times of the year where the background state is less stable and the development of ENSO is favored through a coupled instability mechanism) (Neelin et al. 2000; Kleeman 2008; Stein et al. 2014). Simple models provide partial insight into this instability mechanism by varying one or a few parameters of the background state seasonally. On a side note, some studies even argue that ENSO frequency locking to the seasonal cycle is possible for sufficiently nonlinear resonance (Jin et al. 1994; Tziperman et al. 1994), although they do not deal with the specific physical mechanisms of those interactions.

A great diversity of processes may be responsible for the seasonal synchronization of ENSO in nature. For example, several studies emphasize seasonal changes in the remote wind stress response to SSTs (or Bjerknes feedback) that maintains an east-west asymmetry across the equatorial Pacific (Zebiak and Cane 1987; Jin et al. 2006), as a result for example from the seasonal motion of the intertropical convergence zone (Tziperman et al. 1997), the southward shift of zonal winds in spring (Lengaigne et al. 2006; Stuecker et al. 2013), or the quasi-biennial winds in the far western Pacific (Clarke and Shu 2000). The seasonal variations of wind bursts activity that result among others from the occurrence of the Madden-Julian oscillation (MJO) in boreal winter (Majda and Stechmann 2009; Puy et al. 2016) may also be important for ENSO seasonal synchronization, although this relationship has not been clearly evidenced in modeling studies (Hendon et al. 2007; Seiki and Takayabu 2007; Lopez and Kirtman 2014). Finally, seasonal changes in ocean processes such as the background upwelling and thermocline retroaction on SST in the eastern Pacific have also been documented (Hirst 1986; Galanti et al. 2002), although they are argued by some to be of secondary importance for ENSO (Tziperman et al. 1997).

In addition to the above processes, current theory emphasizes the role of convective activity and cloud cover as a main contender for explaining the ENSO seasonal synchronization (Zebiak and Cane 1987; Jin et al. 2006; Dommenget and Yu 2016). The convective response to ENSO SST anomalies in the tropical $\mathrm{Pa}$ cific can significantly affect the SST in return through exchanges in radiative and turbulent heat fluxes, mainly incoming solar shortwave radiation as well as outgoing latent heat flux (Lloyd et al. 2012; Frenkel et al. 2015). This so-called cloud radiative feedback usually tends to dampen the ENSO variability, as was analyzed in a number of studies (e.g., Waliser et al. 1994; Wang and McPhaden 2001; Dommenget et al. 2014), but is however strongly state dependent and seasonal. The state dependency of the cloud radiative feedback is particularly significant over the eastern Pacific cold tongue region where seasonal changes in the background SSTs and cloud cover are marked. Generally speaking, damping by the cloud radiative feedback in that region tends to be maximal in spring (with warmer SSTs) and minimal in fall (with cooler SSTs) as a result of an ensemble of complex underlying processes. For instance, it has recently been shown in idealized settings that realistic seasonal synchronization of ENSO can result from this simple seasonal dependency (Dommenget and Yu 2016). Despite insight gained from simple models, representing the cloud radiative feedback in CGCMs still presents a major challenge. In fact, such models have significant problems in capturing the variability associated with organized tropical convection despite its dominant role in setting the ENSO characteristics (Guilyardi et al. 2009; Bellenger et al. 2014). The simulation of marine boundary layer clouds or incoming shortwave radiation in the eastern tropical Pacific, for example, remains a major source of uncertainties (Bony and Dufresne 2005; Lloyd et al. 2012).

Recently, a simple stochastic dynamical model was developed that automatically captures the ENSO diversity and intermittency in nature. This ENSO model has been systematically studied in Thual et al. (2016), Chen and Majda (2016a,b), and Chen et al. (2017). It succeeds in recovering the eastern Pacific (EP) moderate and occasional super El Niño with realistic buildup and shutdown of wind bursts (Thual et al. 2016), as well as the central Pacific (CP) El Niño (Chen and Majda 2016a). Importantly, both the variance and nonGaussian statistical features in different Niño regions spanning from the western to the eastern Pacific are captured by the coupled model (Chen and Majda 2016b). The model dynamics, amenable to detailed analysis, consist of state-dependent stochastic wind bursts coupled to simple ocean-atmosphere processes that are otherwise deterministic, linear, and stable, as well as nonlinear advection of SST that facilitates the 
occurrence of the CP El Niño. Such a coupled model where the external wind bursts plays the role of maintaining the ENSO (Moore and Kleeman 1999; Fedorov 2002; Philander and Fedorov 2003; Kleeman 2008) is fundamentally different from the Cane-Zebiak (Zebiak and Cane 1987) and other nonlinear models relying on internal instability (e.g., Jin et al. 1994; Tziperman et al. 1994; Chen et al. 2015).

In the present article, we analyze the seasonal synchronization of ENSO with the incorporation of a cloud radiative feedback in the ENSO model from Thual et al. (2016) and Chen and Majda (2016a,b). For instance, given the realism of such a model and its potential implications for studying ENSO diversity and mechanisms, its capacity to capture major aspects of the ENSO seasonal synchronization in nature is an important requirement. We will show hereafter that, in addition to the above features, the model simulates a realistic chronology for both the EP and the CP El Niño as well as La Niña, with notably a tendency to peak in boreal winter as well as decreased predictability in spring, consistent with the persistence barrier in nature. This goal is achieved thanks to the incorporation of a cloud radiative feedback in the model that accounts for the seasonal variations in convective activity and cloud cover discussed above in simple fashion (Frenkel et al. 2015). Importantly, this cloud radiative feedback is derived here naturally from the model's atmosphere dynamics with no ad hoc assumptions: in particular, a simple collective representation of convective processes is considered in the model's atmosphere that has been successful in other settings to realistically capture the most salient features of the MJO and intraseasonal variability in the tropics (Majda and Stechmann 2009, 2011; Thual et al. 2014; Stechmann and Majda 2015). Finally, other seasonal feedbacks are also potentially important for seasonal synchronization as discussed above and their incorporation in the ENSO model is also documented for completeness. However, those additional seasonal feedbacks show various deficiencies in the present ENSO model as compared to the cloud radiative feedback such as ad hoc formulations, lack of robustness, or observational analogues.

The article is organized as follows. In Section 2, we present the model and its setup including the formulation of the cloud radiative feedback. This section also includes a brief introductory illustration of the ENSO seasonal synchronization in observations. In section 3 we show results from numerical experiments where the role of the cloud radiative feedback on ENSO seasonal synchronization is evidenced. We also document the incorporation of other possible seasonal feedbacks in the model at the end of the section. Section 4 is a discussion with concluding remarks. Additional details on the model are provided in the appendixes.

\section{Model and methods}

\section{a. ENSO seasonal synchronization in observations}

We present here a brief illustration of the ENSO seasonal synchronization in observations as a general guideline and motivation for the model parameterization in the next sections. Figures $1 \mathrm{a}-\mathrm{e}$ show observed El Niño composites as a function of the month of the year for the recent period. Datasets are taken from the NCEP-NCAR, OISST and NCEP GODAS daily reanalyses over 1982-2016 respectively for the $850-\mathrm{hPa}$ zonal winds, SST, and thermocline depth while outgoing longwave radiation (OLR) is provided by the NOAA interpolated OLR monthly dataset over 1982-2013 (Kalnay et al. 1996; Reynolds et al. 2007; Behringer et al. 1998; Liebmann and Smith 1996). Composites are computed from the El Niño events of 1983, 1987, 1992, 1998, 2003, 2010, and 2016 provided data are available. Note that the major El Niño events of 1983, 1998, and 2016 contribute to a large extent to the composites in Fig. 1, and that each individual El Niño event shows unique features beyond the composite (e.g., with westward instead of eastward propagations of SST as well as distinct dominant processes).

As shown in Figs. 1a-e, the ENSO seasonal synchronization is observed on the entire circulation of the equatorial Pacific. El Niño events typically start with increased SST, thermocline depth, zonal winds, and wind burst activity in the western Pacific around boreal spring of the preceding year [Mar(0)]. During summer and fall those anomalies propagate to the central-eastern Pacific where they intensify, eventually reaching their peak in winter $[\operatorname{Dec}(0)]$. A reversal of conditions toward La Niña then initiates around the following spring [Mar(1)]. Although the wind burst activity in Fig. 1a measures the overall amplitude of wind bursts (both westerly and easterly), wind bursts during the development of El Niño events are dominantly westerly (not shown). The OLR inversely measures the overall increased convective activity and upper cloud cover that follow the warm SSTs during their eastward propagation. Hereafter we discuss the role of inverse OLR (-OLR) for clarity.

This seasonal synchronization indicates underlying ENSO dynamics that are state dependent (i.e., directly related to changes in the climatological background 
(a) Wind Bursts

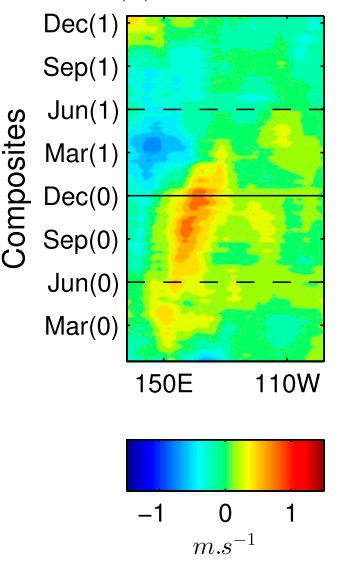

(f) Wind Bursts

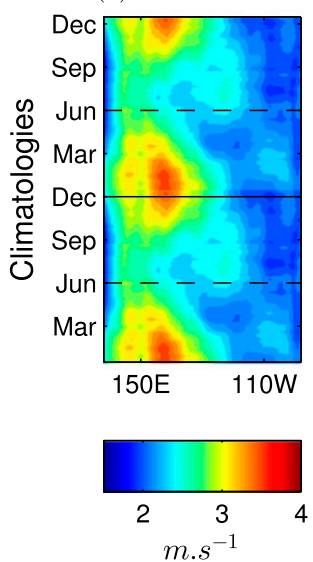

(b) Zonal Winds
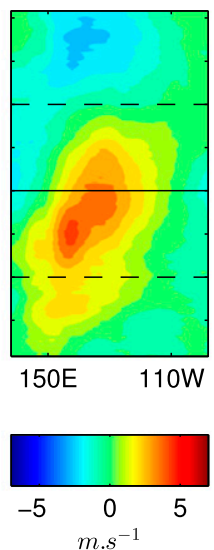

(g) Zonal Winds
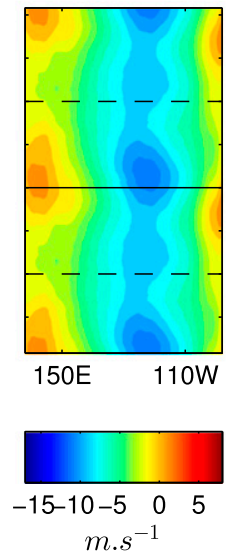

(c) SST

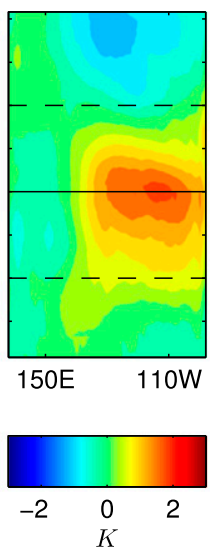

(h) SST

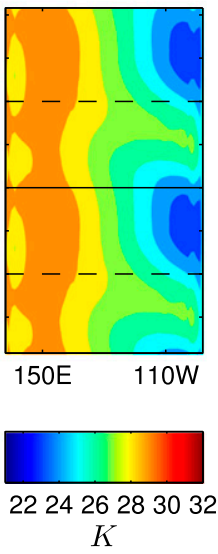

(d) Thermocline
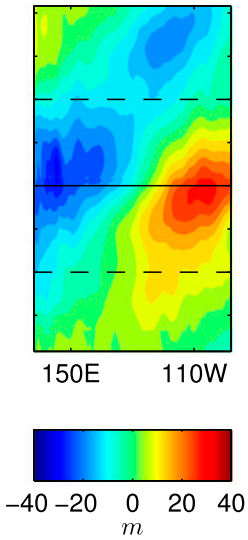

(i) Thermocline
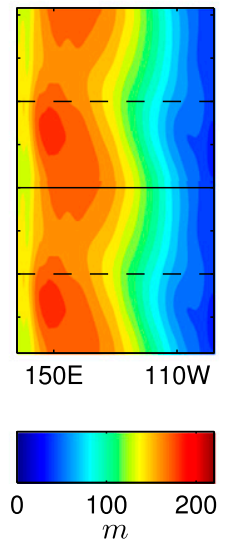

(e) -OLR
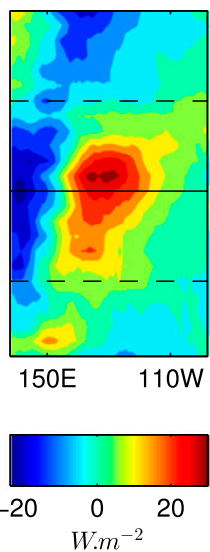

(j) -OLR

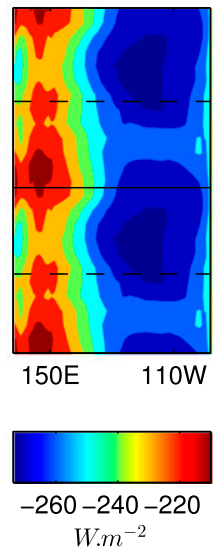

FIG. 1. El Niño composites, as a function of longitude and month of the year for observed anomalies of (a) zonal wind burst activity [90-day running standard deviation (std), $\mathrm{m} \mathrm{s}^{-1}$ ], (b) zonal winds $\left(\mathrm{m} \mathrm{s}^{-1}\right.$ ), (c) SST (K), (d) thermocline depth $(\mathrm{m})$, and (e) - OLR $\left(\mathrm{W} \mathrm{m}^{-2}\right)$, averaged within $5^{\circ} \mathrm{N}-5^{\circ} \mathrm{S}$. (f)-(j) As in (a)-(e), but for associated climatologies.

conditions of the equatorial Pacific), as also summarized in Figs. 1f-j. In the eastern Pacific, the most salient feature is the pronounced climatological SST cooling in boreal fall and warming in spring, as a result of the seasonal motion of the ITCZ and its modification of the upwelling and meridional advection strength (Mitchell and Wallace 1992). Note that the cool SSTs in fall in the eastern Pacific coincide with decreased convective activity and upper cloud cover as measured here by the decreased -OLR. The climatology is fundamentally different in the central to western Pacific, with weak SST variations but increased trade winds in winter and spring due to the intensification of the Walker circulation as well as increased wind burst activity as a direct response to increased atmospheric intraseasonal variability (Hendon et al. 2007; Seiki and Takayabu 2007). Finally, note that although interannual variations of thermocline depth are pronounced their climatological variations are weak.

\section{b. Coupled ENSO model}

We present here the ENSO model used in the article. This ENSO model has been systematically studied in Thual et al. (2016), Chen and Majda 2016a,b, and Chen et al. (2017). It succeeds in recovering the traditional El Niño and occasional super El Niño in the eastern Pacific with realistic buildup and shutdown of wind bursts (Thual et al. 2016), as well as the central Pacific El Niño (Chen and Majda 2016a). Importantly, both the variance and non-Gaussian statistical features in different Niño regions spanning from the western to the eastern Pacific are captured by the coupled model (Chen and Majda 2016b). Additional details on the model are provided in the appendixes, including the definition of all variables, units, and parameter values.

The ENSO model consists of a nondissipative atmosphere coupled to a simple shallow-water ocean and SST budget: 
(a) $\eta$

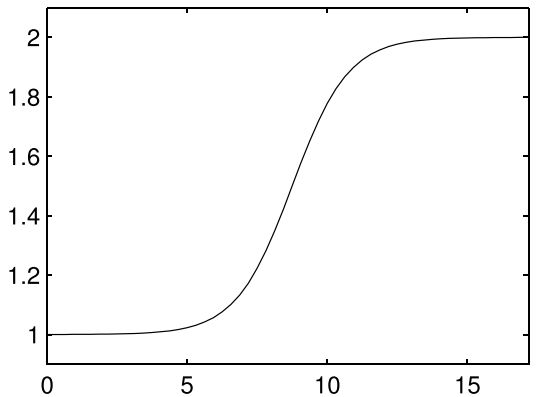

(b) $s_{p}$

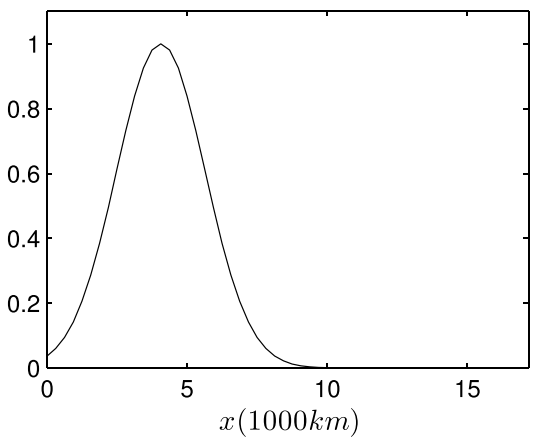

(c) $\alpha(x, \tau)$

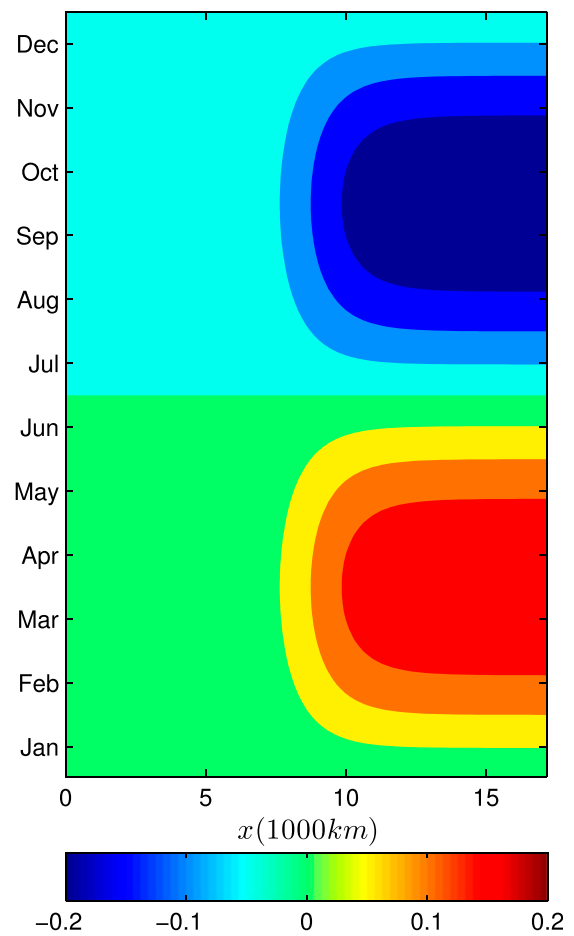

FIG. 2. Model parameterization. Zonal profile of nondimensional (a) thermocline feedback $\eta(x)$ and (b) wind burst structure $s_{p}(x)$ at equator, as a function of zonal position $x$ in $1000 \mathrm{~km}$. (c) Cloud radiative feedback parameter $\alpha(x, \tau)$, as a function of zonal position $x$ and month of the year.

Interannual atmosphere model

$$
\begin{aligned}
-y v-\partial_{x} \theta & =0 \\
y u-\partial_{y} \theta & =0 \\
-\left(\partial_{x} u+\partial_{y} v\right) & =E_{q} /(1-\bar{Q}),
\end{aligned}
$$

Interannual ocean model

$$
\begin{aligned}
\partial_{\tau} U-c_{1} Y V+c_{1} \partial_{x} H & =c_{1} \tau_{x} \\
Y U+\partial_{Y} H & =0 \\
\partial_{\tau} H+c_{1}\left(\partial_{x} U+\partial_{Y} V\right) & =0, \quad \text { and }
\end{aligned}
$$

Interannual SST model

$$
\partial_{\tau} T+\mu \partial_{x}(U T)=-c_{1} \zeta E_{q}+c_{1} \eta H-c_{1} \alpha T,
$$

with

$$
\begin{aligned}
& E_{q}=\alpha_{q} T \text { and } \\
& \tau_{x}=\gamma\left(u+u_{p}\right) .
\end{aligned}
$$

In the above model, $x$ is the zonal direction, $y$ and $Y$ are the meridional direction in the atmosphere and ocean, respectively, and $\tau$ is interannual time. For the atmosphere, $u$ and $v$ are zonal and meridional winds, $\theta$ is potential temperature, and $E_{q}$ is latent heating. For the ocean, $U$ and $V$ are zonal and meridional currents, $H$ is thermocline depth, $\tau_{x}$ is zonal wind stress, and $T$ is SST. All those variables are anomalies from an equilibrium state, and are nondimensional. The term $u_{p}$ is a stochastic wind burst perturbation, as described hereafter. The atmosphere extends over the entire equatorial belt $0 \leq x \leq L_{A}$ with periodic boundary conditions while the Pacific Ocean extends from $0 \leq$ $x \leq L_{O}$ with reflection boundary conditions. Only a few thermodynamic feedbacks deemed most important are retained in the SST budget from Eq. (3), such as dissipation and latent heat losses as well as the effect of nonlinear zonal advection and the thermocline feedback (An and Jin 2001; Thual et al. 2016; Chen and Majda 2016a). The thermocline feedback $\eta(x)$ is maximal in the eastern Pacific, as shown in Fig. 2a. Note that the SST budget in Eq. (3) has been slightly modified as compared to previous works in order to reflect more clearly the breakdown of dissipative processes, including the cloud radiative feedback of intensity $\alpha$ described hereafter. 
The above ENSO model introduces several unique theoretical elements. First, without stochastic wind bursts $u_{p}$ and nonlinear zonal advection of SST the resulting coupled system is linear, deterministic, and stable. Such a coupled model where the external wind bursts play the role of maintaining the ENSO (Moore and Kleeman 1999; Philander and Fedorov 2003; Kleeman 2008) is fundamentally different from the Cane-Zebiak (Zebiak and Cane 1987) and other nonlinear models (e.g., Jin et al. 1994; Tziperman et al. 1994; Chen et al. 2015) relying on internal instability. Second, a nonlinear zonal advection of SST is adopted in Eq. (3) that facilitates the intermittent occurrence of the central Pacific El Niño with realistic features (Chen and Majda 2016a). This nonlinear zonal advection involves the contribution from both mean and fluctuation, which differs from previous work that relies on linear advection only and requires ad hoc parameterization of the background SST gradient (e.g., Dewitte et al. 2013). Third, instead of a Gill-type atmosphere (Gill 1980) the atmosphere is here nondissipative and consistent with the skeleton model for the MJO (Majda and Stechmann 2009, 2011), valid here on the interannual time scale and suitable to describe the dynamics of the Walker circulation (Majda and Klein 2003; Stechmann and Ogrosky 2014; Stechmann and Majda 2015). Finally, note that the meridional axes $y$ and $Y$ are different in the atmosphere and ocean as they each scale to a suitable Rossby radius. This allows for a systematic meridional decomposition of the system into the well-known parabolic cylinder functions (Majda 2003), which keeps the system low-dimensional [not shown; see the supplemental information of Thual et al. (2016)].

\section{c. Stochastic wind burst model}

Stochastic wind bursts perturbations are added to the model that represent several important ENSO triggers found in nature such as westerly wind bursts (WWBs) and easterly wind bursts (EWBs) as well as the convective envelope of the MJO (Thual et al. 2016). The wind bursts activity is driven here by a simple stochastic process that accounts for its irregular, intermittent, and unpredictable nature on the interannual time scale as well as its dependence on the western Pacific warm pool strength.

The wind bursts perturbations in Eq. (4) are:

$$
u_{p}=a_{p}(\tau) s_{p}(x, y)
$$

with fixed spatial structure $s_{p}$ centered in the western Pacific as shown in Fig. 2b, and amplitude $a_{p}$ (positive for a WWB and negative for an EWB) that evolves as follows:

$$
\frac{d a_{p}}{d \tau}=-d_{p}\left(a_{p}-\hat{a}_{p}\right)+\sigma_{p} \dot{W}(\tau),
$$

where $d_{p}$ is dissipation, $\hat{a}_{p}$ is a mean trade winds strengthening, and $\dot{W}$ is a white noise source of intensity $\sigma_{p}$. The mean trade winds strengthening $\hat{a}_{p}<0$ accounts for the occasional intensification of the Walker circulation on decadal time scales, as observed for example in recent decades (Chen and Majda 2016a). The noise source intensity $\sigma_{p}$ and mean trade winds strengthening $\hat{a}_{p}$ are here state-dependent on the western Pacific warm pool strength, as described hereafter.

\section{d. Three-state Markov jump process}

The characteristics of wind burst activity in nature can change dramatically depending on the state of the equatorial Pacific, for example with a rapid buildup and shutdown during El Niño events as well as distinct features for El Niño SST anomalies either in the eastern or central Pacific. To model this behavior in a simple fashion, the characteristics of wind burst activity are here state-dependent and evolve according to a simple Markov jump process with three states (Gardiner 1994; Lawler 2006; Majda and Harlim 2012).

First, we allow the equatorial Pacific system to switch back and forth between three states $s=0,1,2$ with different wind burst characteristics in Eq. (7):

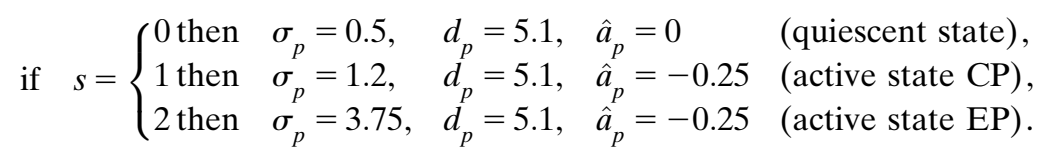

The quiescent state 0 accounts for the conditions in the absence of El Niño activity or during La Niña with weak wind burst activity $\sigma_{p}$ and no mean trade winds strengthening $\hat{a}_{p}$. The active state 1 accounts for the conditions during periods with central Pacific El Niño events with moderate wind burst activity and enhanced mean trade winds (e.g., as observed in the 1990s). The active state 2 accounts for the conditions during traditional eastern Pacific El Niño events with strong wind burst activity as well as enhanced mean trade winds kept for consistency with the other active state 1 . The dissipation rate $d_{p}$ (around 6.7 days) is identical in each state.

Second, we allow for intermittent transitions between the three states $s=0,1,2$ depending on the strength of 
the equatorial Pacific warm pool. The probabilities of transiting from a given state $i$ to another state $j \neq i$ or to remain in the same state $i$ after a time interval $\Delta \tau$ are

$$
\begin{aligned}
& P[s(\tau+\Delta \tau)=j \mid s(\tau)=i]=\mu_{i j} \Delta \tau+o(\Delta \tau), \\
& P[s(\tau+\Delta \tau)=i \mid s(\tau)=i]=1-\sum_{i \neq j} \mu_{i j} \Delta \tau+o(\Delta \tau) .
\end{aligned}
$$

Importantly, the transition rates $\mu_{i j}$ are state-dependent on the warm pool strength, that is, on $T_{W}$, the average of SST anomalies in the western half of the equatorial Pacific $\left(0 \leq x \leq L_{O} / 2\right)$, according to a simple general relationship:

$$
\begin{aligned}
& \mu_{i j}=\left[1+\tanh \left(2 T_{W}\right)\right] / r_{i j} \text { if } \quad i<j, \\
& \mu_{i k}=\left[1-\tanh \left(2 T_{W}\right)\right] / r_{i k} \text { if } \quad i>k,
\end{aligned}
$$

with coefficients $r_{i j}$ provided in the Table A2. A stronger warm pool $\left(T_{W} \geq 0\right)$ favors the transition to higher states with increased wind burst activity, and conversely a weaker warm pool $\left(T_{W} \leq 0\right)$ favors the transition to lower states with decreased wind burst activity. For instance, wind bursts of increased intensity and zonal fetch are usually favored by warmer SSTs in the western Pacific or when the warm pool extends eastward (Hendon et al. 2007; Seiki and Takayabu 2007; Puy et al. 2016), which is accounted for here in a simple fashion through changes in $T_{W}$. In particular, this state dependence of stochastic wind burst activity on western Pacific SST is fundamentally different from the one of other models (Jin et al. 2007) that relies on the eastern Pacific SST and in addition requires no ad hoc prescription of wind bursts thresholds and propagation (Chen et al. 2015).

\section{e. Seasonal cloud radiative feedback}

We now introduce the cloud radiative feedback used in the above ENSO model. The cloud radiative feedback is derived naturally from the model formulation with no ad hoc assumptions and accounts for seasonal variations in cloud cover and convective activity in the eastern Pacific in simple fashion.

First, we briefly recall the derivation of the atmosphere in the above ENSO model. The atmosphere is here nondissipative and consistent with the skeleton model for the MJO (Majda and Stechmann 2009, 2011). The starting skeleton model reads as follows:

$$
\begin{aligned}
\partial_{t} u-y v-\partial_{x} \theta & =0 \\
y u-\partial_{y} \theta & =0 \\
\partial_{t} \theta-\left(\partial_{x} u+\partial_{y} v\right) & =\bar{H} a-s^{\theta} \\
\partial_{t} q+\bar{Q}\left(\partial_{x} u+\partial_{y} v\right) & =-\bar{H} a+s^{q}+E_{q} \\
\partial_{t} a & =\Gamma q a,
\end{aligned}
$$

where, in addition to the variables described in Eq. (1), $t$ is intraseasonal time, $q$ is lower-level moisture anomalies, and $a \geq 0$ is the planetary envelope of convective activity. The quantities $s^{\theta}$ and $s^{q}$ are prescribed external background sources of cooling and moistening, respectively. As compared to Majda and Stechmann (2009) the moisture budget for $q$ has here been extended with the contribution of latent heat release $E_{q}$ to account for coupling with the ocean. In particular, the planetary envelope $a$ is a collective (i.e., integrated) representation of the convection/ wave activity occurring at small scales, the details of which are unresolved. For instance, a broad range of convective events occurs in the tropical Pacific with varying effects on the dynamical and thermodynamic properties of the atmosphere. Their collective effect on the planetary scale and their dependency on lowlevel moisture is accounted for in a simple fashion through $a$ in the skeleton model. In Eq. (10) convective activity heats and dries the atmosphere at the same time through $\pm \bar{H} a$ and tends to develop for moist conditions at rate $\Gamma$. This simple parameterization allows the skeleton model to realistically capture the most salient features of the MJO and intraseasonal variability in the tropics (Majda and Stechmann 2009, 2011; Thual et al. 2014; Stachnik et al. 2015). In particular, -OLR, which measures overall changes in convective activity and upper cloud cover in nature (see, e.g., Figs. $1 \mathrm{e}$ and $1 \mathrm{j}$ ) is a direct observational analogue (i.e., surrogate) for $a$ in the model (up to a constant multiplier; Stechmann and Majda 2015).

Second, while the skeleton model is originally intended at intraseasonal variability it has been shown to describe realistic dynamics of the Walker circulation relevant to ENSO in the asymptotic limit of interannual fluctuations (Majda and Klein 2003; Stechmann and Ogrosky 2014; Ogrosky and Stechmann 2015). Replacing intraseasonal time $t$ with interannual time $\tau=\varepsilon t$ in Eq. (10) with $\varepsilon$ small and retaining the first order of the expansion into powers of $\varepsilon$, we obtain

$$
\begin{aligned}
\bar{H}(a-\bar{a}) & =E_{q} /(1-\bar{Q}), \\
\bar{H} \bar{a} & =\left(s^{q}-\bar{Q} s^{\theta}\right) /(1-\bar{Q}),
\end{aligned}
$$

as well as the interannual atmosphere model from Eq. (1). In particular, convective activity $a$ increases with latent heat $E_{q}$ released in the atmosphere, while its background value $\bar{a}$ results from the adjustment to the external sources $s^{\theta}$ and $s^{q}$. The above asymptotic expansion is detailed in appendix B [see also the supplemental information of Thual et al. (2016)]. 
Third, we account for the effect of convective activity $a$ on the underlying SSTs as follows:

$$
\partial_{\tau} T+\mu \partial_{x}(U T)=-c_{1} \zeta E_{q}+c_{1} \eta H-c_{1} \alpha_{c}(a-\bar{a}),
$$

where increased convective activity $a$ simply acts as a dissipation term with intensity $\alpha_{c}$. Again, this accounts for the collective effect of several types of convective events and their radiative and turbulent heat flux exchanges with the ocean. For example, low and thick clouds, such as congestus, primarily reflect solar radiation and cool the surface whereas high, thin clouds, such as stratiform clouds, transmit some of the incoming solar radiation and reflect back some of the outgoing longwave radiation (Frenkel et al. 2015). Modifying the expression of convective activity in the SST budget from Eq. (12) using Eq. (11) as well as Eq. (4), which relates latent heat to SST (i.e., $E_{q}=\alpha_{q} T$ ), we retrieve the SST budget from Eq. (3):

$$
\partial_{\tau} T+\mu \partial_{x}(U T)=-c_{1} \zeta E_{q}+c_{1} \eta H-c_{1} \alpha T,
$$

where $\alpha=\alpha_{q} \alpha_{c}$. The cloud radiative feedback is the last term on the rhs, acting as an SST dissipation term for $\alpha \geq 0$.

Finally, seasonal variations of the cloud radiative feedback in the ENSO model are accounted for as follows. We vary $\alpha(x, \tau)$ as a function of zonal position and month of the year as shown in Fig. 2c. Those variations are overall consistent with the literature (Lloyd et al. 2012; Frenkel et al. 2015; Dommenget and Yu 2016) and reflect changes in the observed climatology of SST shown in Fig. 1h in simple fashion. In fall when the eastern Pacific region is dominated by cool background SSTs, subsidence and decreased cloud cover there is overall a weak convective response to SST anomalies as usually only a few clouds are created or destroyed. An SST warming can even in some case break the low-level cloud cover by destabilizing the atmospheric boundary layer. This is evident in Figs. 1c and 1e where in the fall season El Niño composites show a weak increase in overall convective activity (-OLR) in the eastern Pacific despite a strong SST increase in that region. As a result, the SST damping by the cloud radiative feedback is weak as reflected by $\alpha$ being minimal. In spring when the eastern Pacific is dominated by warm background SSTs and ascents the convective response to SST warming is much more significant with increased upperlevel cloud cover and decreased incoming shortwave radiations. As a result, the SST damping by the cloud radiative feedback is strong as reflected by $\alpha$ being maximal. Meanwhile, there are no seasonal variations of the cloud radiative feedback in the western Pacific where the SST climatology is weak. For the present parameter values the SST dissipation rate from the cloud radiative feedback varies seasonally within reasonable range $\pm(0.9 \mathrm{yr})^{-1}$ in the eastern Pacific. In addition, for simplicity the variations are sinusoidal with a zero background time mean of $\alpha$ (which can be absorbed in $\zeta$ ) such that we retrieve on average the dynamics from previous work (Thual et al. 2016; Chen and Majda 2016a,b).

\section{Model properties}

In this section, we show results from numerical experiments with the ENSO model described above. Despite the model simplicity, the main features of ENSO seasonal synchronization in nature are captured qualitatively. In addition to simulating the ENSO diversity with realistic non-Gaussian statistics in different Niño regions, both the EP moderate and super El Niño, the CP El Niño, and La Niña show a realistic chronology with a tendency to peak in boreal winter as well as decreased predictability in spring consistent with the persistence barrier in nature. The cloud radiative feedback favors the development of those events in fall and their shutdown the following spring, with, however, different contributions for either the EP or CP type of events. We also document the incorporation of other possible seasonal feedbacks in the model at the end of the section.

\section{a. Statistical properties}

Figure 3 summarizes the main statistical properties of the ENSO model as computed from the long-term solutions of a numerical experiment $(5000 \mathrm{yr})$ with the seasonal cloud radiative feedback. Such an experiment is sufficient long with many ENSO events to be statistically reliable (e.g., with small confidence intervals). Figure 3a shows a histogram of El Niño and La Niña events peak as a function of the month of the year, as detected using the index Niño-3.4 SST computed from the model outputs. Such a histogram allows us to assess the seasonal synchronization of ENSO events in the model and has been used previously in the literature (e.g., Tziperman et al. 1997), even though other measures could be used (e.g., Stein et al. 2014). Both El Niño and La Niña events in Fig. 3a tend to peak around November-December at the end of the calendar year, as in nature. In fact, almost all ENSO events in the recent observational record have peaked around December (not shown). Another realistic consequence of this synchronization is the presence of a predictability barrier in boreal spring as in nature (Torrence and Webster 1998; Levine and McPhaden 2015). In Fig. 3b we show a lagged regression of Niño-3.4 SST on itself as a function 
(a) Histogram Nino 3.4 (events/yr)

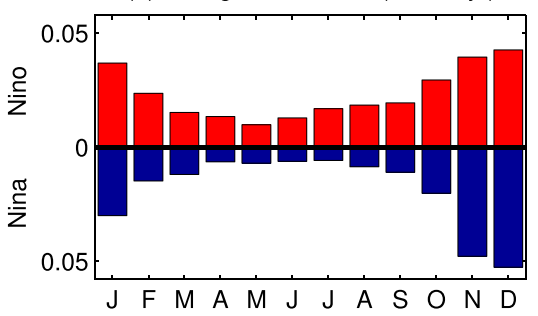

(c) Std Nino3.4

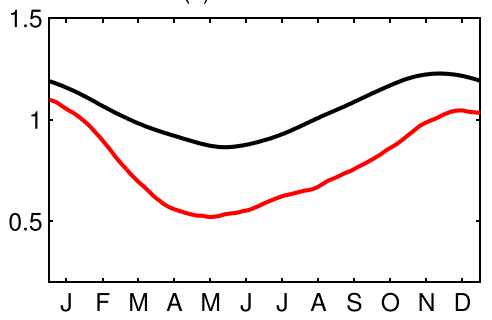

(b) Regression Nino 3.4

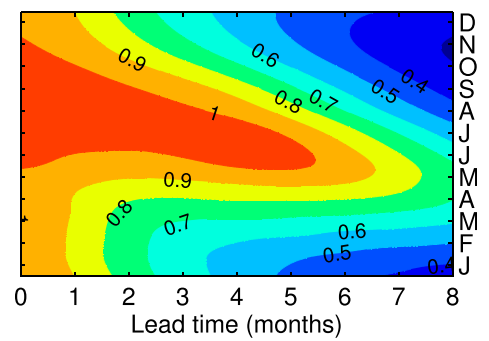

(d) Spectrum Nino3

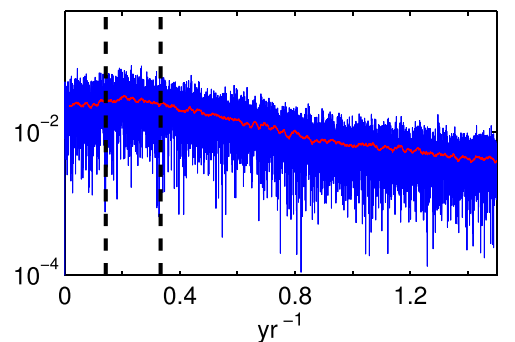

(g) Pdf Nino 3

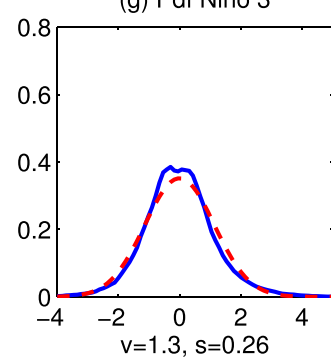

FIG. 3. Model statistics. (a) Histogram of El Niño (red) and La Niña (blue) events peaks (events per year), as a function of month of the year. El Niño events peaks are detected as local maxima with Niño-3.4 SST anomalies $\geq 1 \mathrm{~K}$ ( $\leq-1 \mathrm{~K}$ for La Niña events) with only one El Niño event allowed within a 2-yr period. (b) Regression coefficient of Niño-3.4 SST on itself (in $\mathrm{K} \mathrm{K}^{-1}$ with 0.1 contour interval), as a function of month of the year and lead time. (c) Standard deviation of Niño-3.4 SST as a function of month of the year in the model (black) and observations (red). (d) Power spectrum of Niño-3 SST from the model. Dashed black lines indicate the 3-7-yr band. (e)-(g) PDFs of Niño-4, Niño-3.4 and Niño-3 SST, respectively, from the model with a Gaussian fit (red dashed) and indication of variance $v$ and skewness $s$.

of month of the year and lead time. This illustrates the simplest prediction scheme for Niño-3.4 SST in the model that can be constructed based on linear regressions conditional on seasons. For example, Niño-3.4 SST conditions in February (denoted by F; $y$ axis) with a lead time of 6 months ( $x$ axis) are used to predict Niño3.4 conditions the next August. In addition, regression values are $>1$ for predictions initiated around boreal summer (JJA) due to the increase in standard deviation the next boreal fall and winter. Note that we show here lagged regressions instead of lagged correlations because those are used in practice for predictions (see, e.g., Chen and Majda 2015). The predictability barrier appears in Fig. $3 b$ as a decreased regression coefficient in boreal winter to spring followed by a sharp increase in boreal spring to summer. For example, predictions initiated in February-April and forecasting up to a lead time of 2-7 months show a weaker regression coefficient than predictions initiated the following months of MayJuly with a similar lead time. This is notably consistent with a development of ENSO events and associated SST anomalies initiating in spring in the model, as shown hereafter. Meanwhile the strong SST anomalies in November-December associated to the peaking of ENSO events are predictable up to several months in advance. Figure $3 \mathrm{c}$ shows in addition the standard deviation of Niño-3.4 SST as a function of month of the year. The results agree very well with observations (red line) despite a slightly increased variability. The standard deviation is minimal in boreal spring during the initiation of ENSO events and maximal in winter during their peak. 
In addition to the seasonal synchronization, other important statistical features of the model remain quite consistent with nature, as in previous work (Thual et al. 2016; Chen and Majda 2016a,b). Those features are here briefly summarized to show the model's robustness to the cloud radiative feedback parameter perturbation. Figures $3 \mathrm{e}$ and $3 \mathrm{f}$ show the probability density functions (PDFs) for the SST indices Niño-4, Niño-3.4, and Niño-3 from the model. Consistent with observations, the PDFs show negative and positive skewness in the Niño-4 and Niño-3 regions, respectively, as well as minimal variance in the Niño-4 region. The presence of a fat tail together with the positive skewness in Niño-3 indicates the occasional super El Niño event in the eastern Pacific. Note that Niño-3 skewness is higher in observations though the present 34-yr-long record may be insufficient to form unbiased statistics [see Chen et al. (2017) for a discussion]. Figure $3 \mathrm{~d}$ shows in addition the power spectrum of Niño-3 SST from the model that is distributed rather evenly in the interannual band (3-7yr), as in nature (Kleeman 2008).

The ENSO model also succeeds in recovering the EP moderate and occasional super El Niño with realistic buildup and shutdown of wind bursts as well as the CP El Niño, as in previous works (Thual et al. 2016; Chen and Majda 2016a,b). This is briefly illustrated by the Hovmöller diagrams in Fig. 4. In this example, at $t=3875 \mathrm{yr}$ there is first an isolated moderate EP El Niño followed by a reversal to La Niña conditions. Next, during $t=3878$ $3889 \mathrm{yr}$ there is sequence of moderate CP El Niño events followed by a strong EP El Niño event and La Niña, a situation qualitatively similar for example to the period 1990-2000 in nature. In particular, the strong El Niño EP event starts around $t=3885 \mathrm{yr}$ with increased SST and thermocline depth anomalies in the western Pacific that then propagate and intensify in the central to eastern Pacific at $t=3886 \mathrm{yr}$ in response to a series of strong westerly wind bursts $\left(a_{p} \geq 0\right)$. Finally, during $t=3894-$ $3898 \mathrm{yr}$ there is another sequence of moderate CP El Niño events, a situation qualitatively similar for example to the period 2002-06 in nature. There is also an example at $t=3870 \mathrm{yr}$ in which strong wind bursts do not trigger any El Niño event, showing that wind burst activity in the model is a necessary but nonsufficient condition to $\mathrm{El}$ Niño development.

\section{b. Chronology of El Niño events}

Figure 5 highlights the overall formation mechanisms and chronology of El Niño events in the model, as shown from lagged correlations between Niño-3.4 SST anomalies from the model and other fields. The correlations are statistically significant at the $95 \%$ confidence interval. This chronology is overall in very good agreement with the one deduced from observation composites in Fig. 1. Importantly, the interplay between this chronology and the seasonal cloud radiative feedback is key for the ENSO seasonal synchronization in the model.

The chronology of El Niño events as shown in Fig. 5 can be roughly separated into a buildup, trigger, and shutdown phase. First, during the buildup phase from around -2 to $-0.5 \mathrm{yr}$ prior to the event the peak SST and thermocline depth anomalies gradually increase in the western Pacific, with associated westward winds and currents due to the intensification of the Walker circulation. Although wind bursts are randomly generated, predominantly easterly wind bursts may contribute to this buildup phase as shown by the negative lagged correlation with $a_{p}$ at around $-1 \mathrm{yr}$ (Fedorov 2002). Second, during the trigger phase around $-0.5 \mathrm{yr}$ to the event peak, positive SST and thermocline depth anomalies propagate and intensify in the central to eastern Pacific following the eastward expansion of the warm pool, with associated eastward winds and currents. Wind bursts that are predominantly westerly are essential to this phase (Seiki and Takayabu 2007). Third, during the shutdown phase in the aftermath of the event peak, wind burst activity weakens due to the cooling of the western Pacific [as the system returns to the quiescent Markov state 0 from Eq. (7)], which initiates a gradual reversal of conditions toward a weak La Niña state.

As shown in Fig. 5f, the cloud radiative feedback contributes to the evolution of El Niño events through heating or cooling of the eastern Pacific: this is shown by contouring regressions with the heating/cooling term $-\alpha T$ from Eq. (3) (for which we recall that there is a dependency of $\alpha$ with seasons; see Fig. 2c. Note that in Eq. (3) the main heating/cooling terms are the thermocline feedback and SST dissipation that approximatively cancel, while the term $-\alpha T$ has a smaller contribution (around $25 \%$ of the residual between thermocline and dissipation, depending on the ENSO events). First, during the buildup phase around $-1 \mathrm{yr}$ prior to the event peak the feedback cooling maintains the negative SSTs in the eastern Pacific and the Walker circulation. Second, the largest contribution of the cloud radiative feedback is during the trigger phase from around $-0.5 \mathrm{yr}$ to the event peak with strong heating that intensifies the SST warming in the eastern Pacific. Finally, during the shutdown phase from around +0.2 to $+0.5 \mathrm{yr}$ the feedback cooling damps SST anomalies to some extent. Importantly, this interplay between the seasonal cloud radiative feedback and the chronology of El Niño events is key for the ENSO seasonal synchronization in the model. For instance, recall from Fig. 2c that $\alpha$ is negative in fall, which coincides with the strong 
(a) $u$

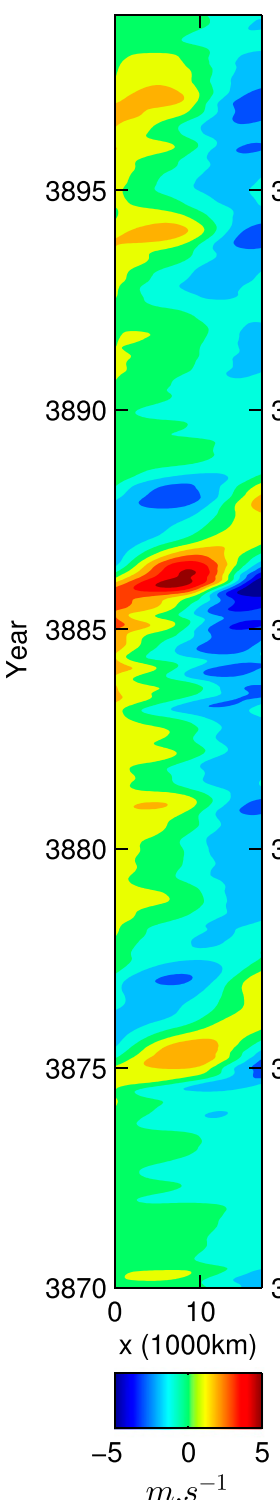

(b) $U$
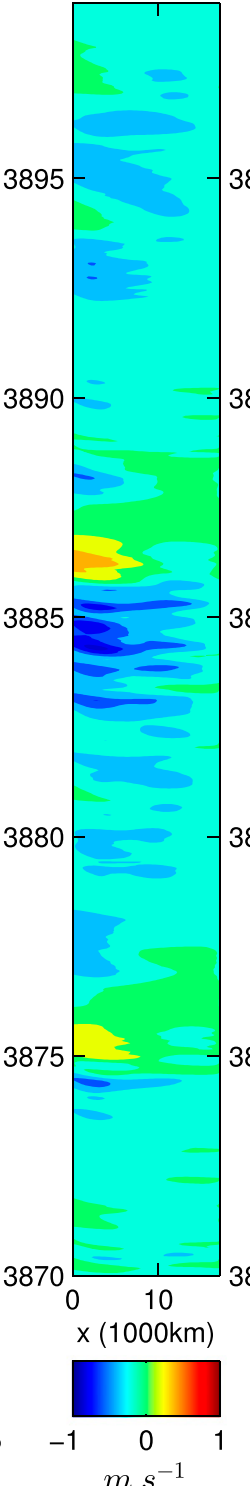

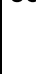

$890-$
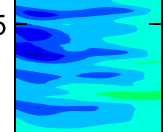

(c) $H$

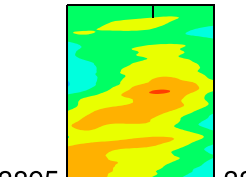

80

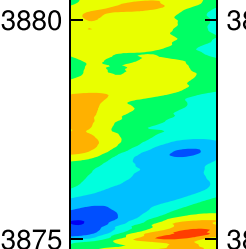

3875
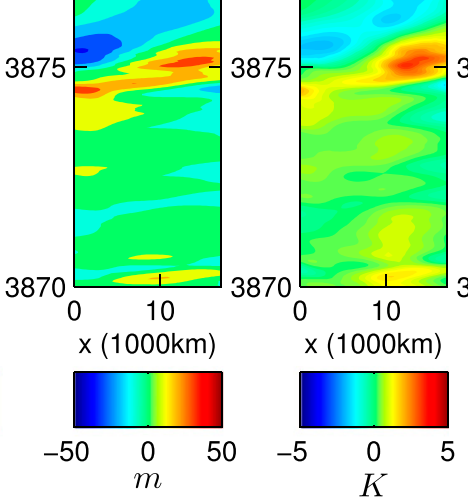

$\mathrm{x}(1000 \mathrm{~km})$
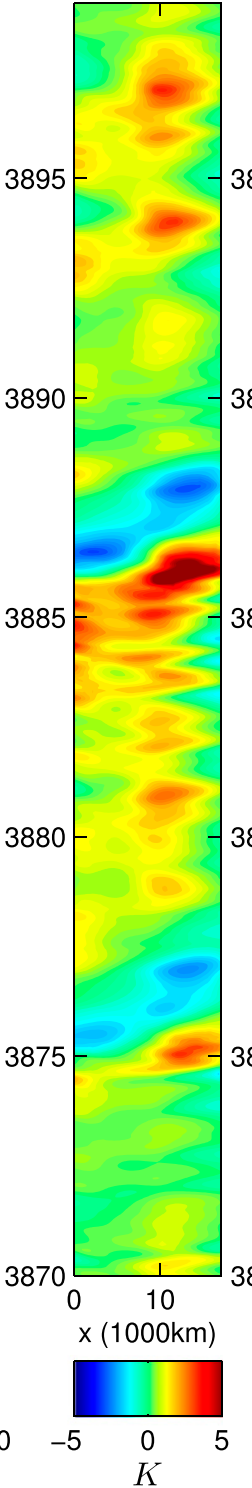

(e) $a_{p}$

(f) Indices

(g) States
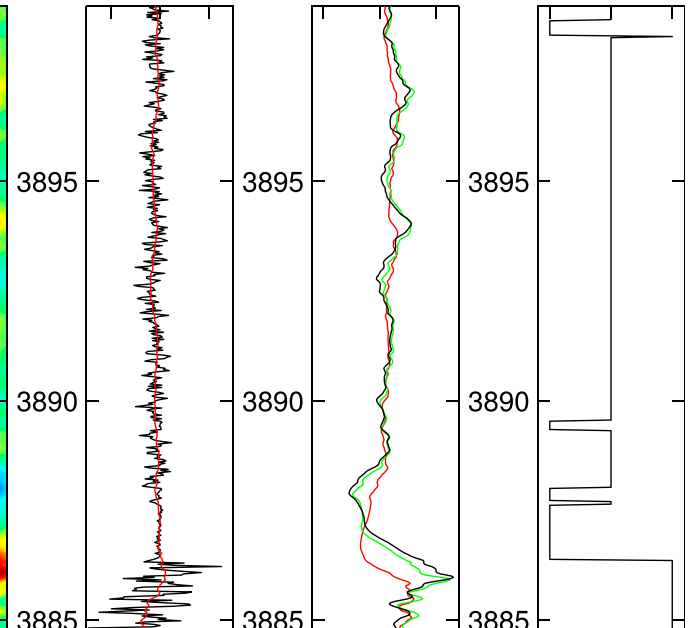

3885

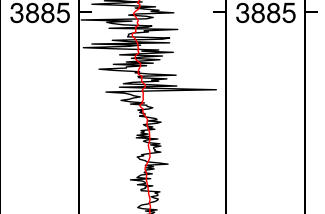

$-3880$

3880
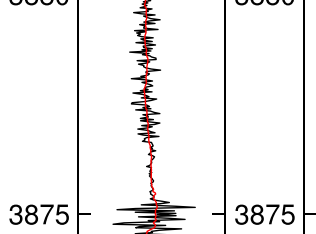

3875
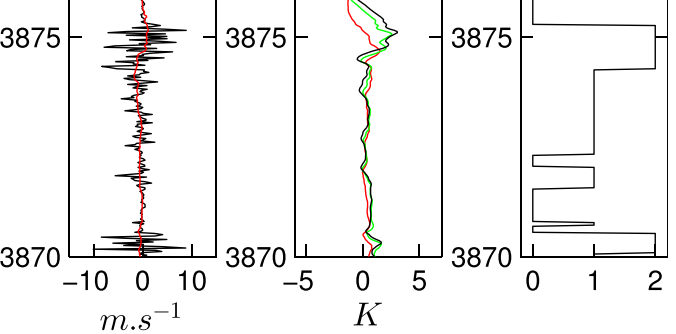

FIG. 4. Hovmöller diagrams with examples of EP moderate and strong El Niño as well as CP El Niño events from the model. Variables at equator as a function of zonal position $x(1000 \mathrm{~km})$ and time (yr): (a) zonal winds $u\left(\mathrm{~m} \mathrm{~s}^{-1}\right)$, (b) zonal currents $U\left(\mathrm{~m} \mathrm{~s}^{-1}\right)$, (c) thermocline depth $H(\mathrm{~m})$, and (d) SST (K). Time series of (e) wind burst activity $a_{p}\left(\mathrm{~m} \mathrm{~s}^{-1}\right.$, including a 120-day running mean in red), (f) SST indices (K) Niño-4 (red), Niño-3.4 (green), and Niño-3 (black), and (g) the state of the Markov process.

trigger phase of El Niño events in Fig. 5, whereas it is positive during the shutdown phase of those events the following spring.

\section{c. Eastern Pacific versus central Pacific El Niño events}

While the present ENSO model succeeds in recovering realistically the moderate and super EP El Niño as well as the CP El Niño, the interplay with the cloud radiative feedback is different for each of those events, leading to slightly modified seasonal synchronization.

To assess this, we analyze here additional experiments where the EP or CP El Niño events are isolated. For instance, in Thual et al. (2016) a simpler model setup is considered that realistically captures the moderate and strong EP El Niño in nature but does not produce the CP El Niño. Similarly, in Chen and Majda (2016a) the simpler setup allows us to capture the CP El Niño but not the EP El Niño. Here we simply reproduce those 

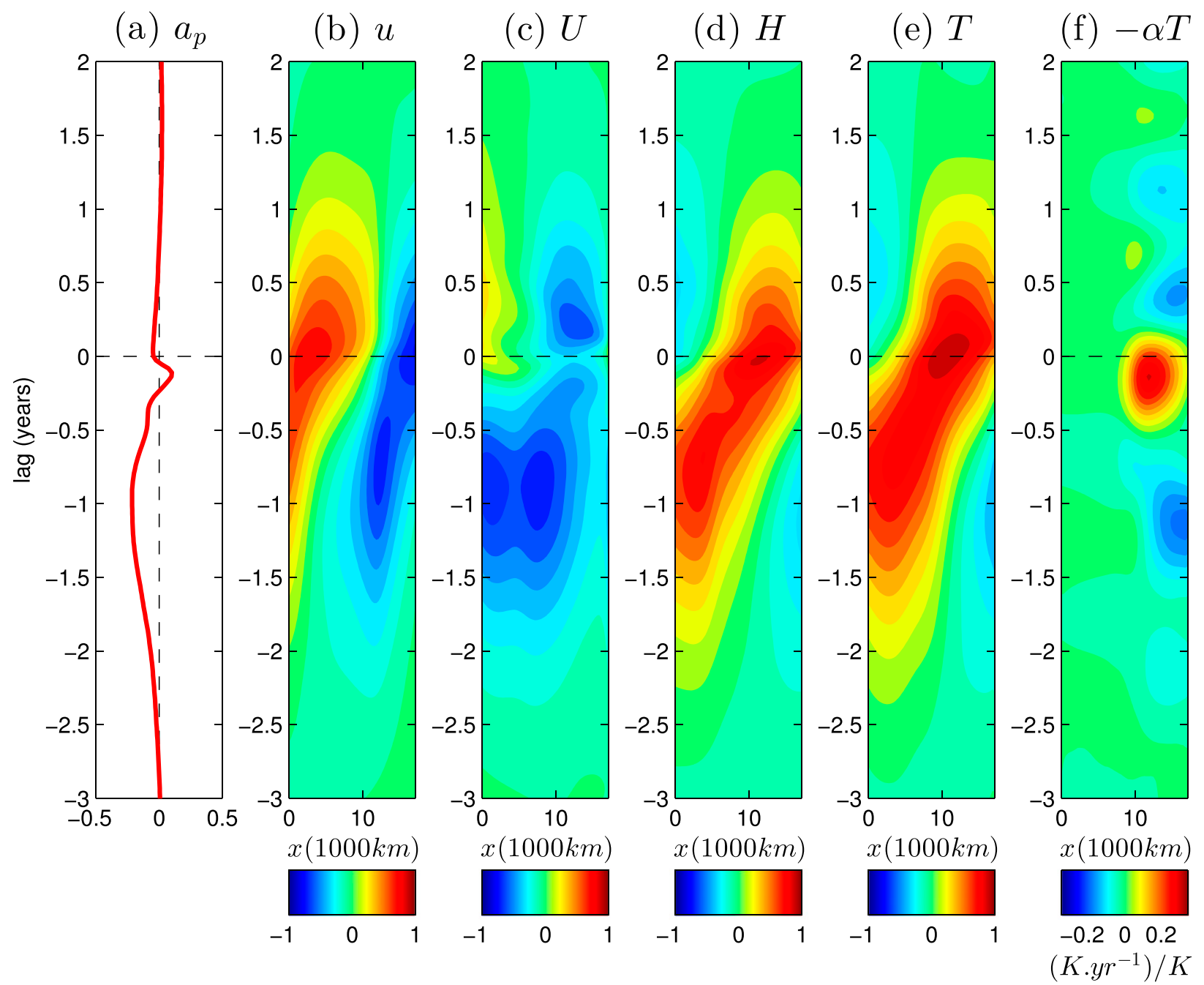

FIG. 5. Chronology of El Niño events in the model. Lagged correlations as a function of zonal position $x(1000 \mathrm{~km})$ and lag time (yr) of Niño-3.4 SST with (a) wind burst amplitude $a_{p}$, (b) zonal winds $u$, (c) zonal currents $U$, (d) thermocline depth $H$, and (e) SST. (f) Lagged regression of the cloud radiative feedback heating $-\alpha T$ on Niño-3.4 SST $\left(\mathrm{K} \mathrm{yr}^{-1} \mathrm{~K}^{-1}\right)$. A positive lag indicates Niño-3.4 SST leads.

experiments with the inclusion of the cloud radiative feedback from the present paper in order to analyze the EP and CP El Niño and their seasonal synchronization independently. The isolated EP El Niño is obtained from the present ENSO model by removing the nonlinear zonal advection $\mu$, mean trade wind strengthening $\hat{a}_{p}$, and active CP state $s=1$ in section 2 while the isolated CP El Niño is obtained by removing the active EP state $s=2$ [along with additional minor modifications; see details in Thual et al. (2016) and Chen and Majda (2016a)]. While we focus here on the seasonal synchronization of isolated EP and CP events for simplicity, note that a more complete analysis should also focus on the interplay and relative occurrence of those events in the complete model (see, e.g., Chen et al. 2017).
Results from the experiments with isolated EP or CP El Niño are summarized in Fig. 6. For brevity and consistency we use the index Niño-3.4 SST to measure the occurrence and intensity of both type of events despite their slightly different localization. As shown in the histograms from Fig. 6, both the isolated EP and CP El Niño as well as their associated La Niña events are realistically synchronized to the seasonal cycle with peaking in boreal winter, as in the complete model. This also evidences the model's robustness to the cloud radiative feedback parameter perturbation. There are, however, slight discrepancies between experiments such as, for example, a decreased occurrence of the isolated EP El Niño due to slightly different model statistics [e.g., a stronger yet rarer super El Niño in the setup from Thual et al. (2016); not shown]. The lagged 
(a) EP Nino: Histogram (events/yr)

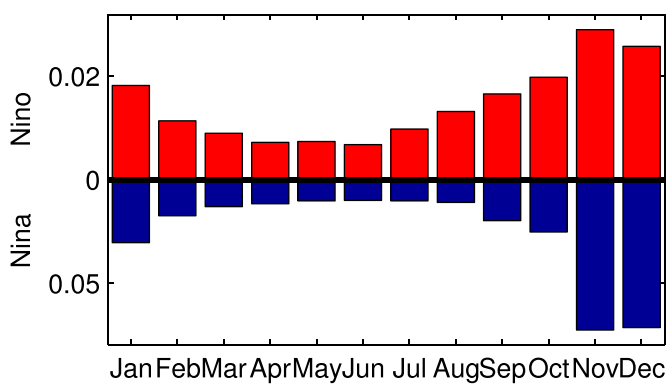

(b) EP Nino: $T$

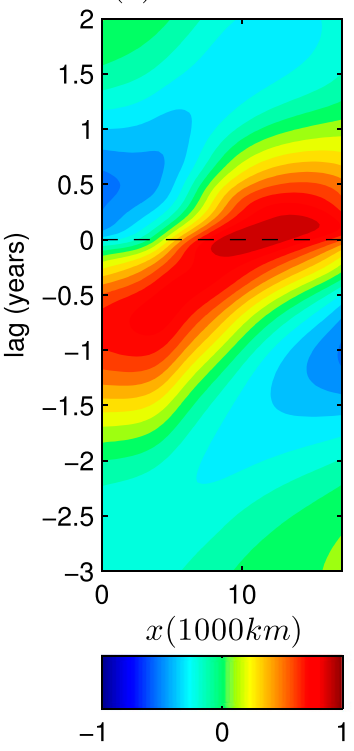

(c) EP Nino: $-\alpha T$
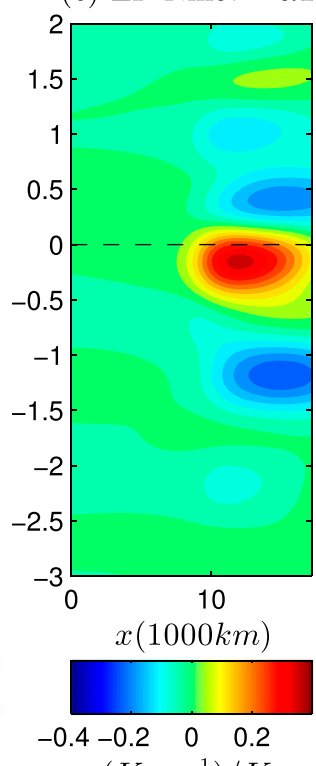

(d) CP Nino: Histogram (events/yr)

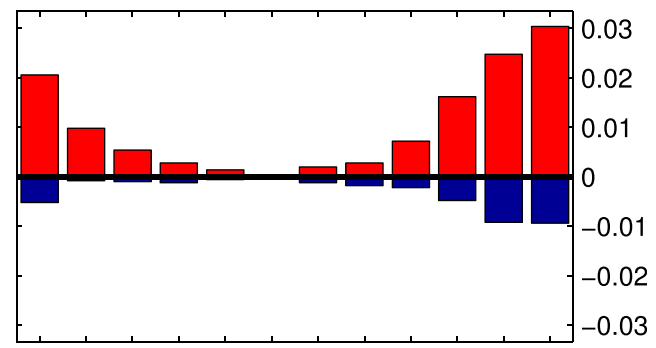

JanFebMarAprMayJun Jul AugSepOctNovDec

(e) CP Nino: $T$
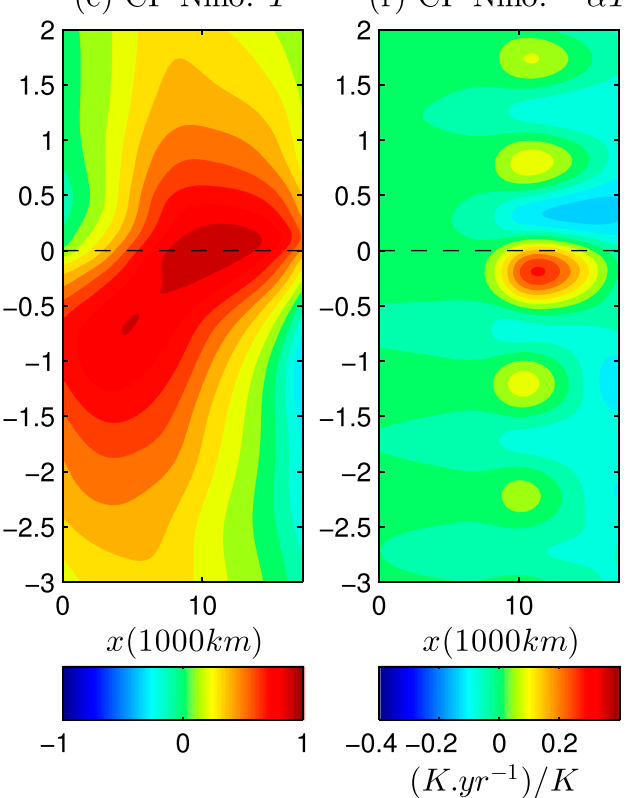

FIG. 6. Experiment with isolated EP El Niño events: (a) histogram of El Niño and La Niña events, (b) lagged correlation of SST, and (c) lagged regression of the cloud radiative feedback heating on Niño-3.4 SST, as in Fig. 5. (d)-(f) As in (a)-(c), but for experiment with isolated CP El Niño events.

correlations and regressions in Fig. 6 highlight the evolution of SST and the cloud radiative feedback heating/cooling during either the isolated EP or CP El Niño. The chronology of the isolated EP El Niño and feedback contribution is similar to the one of the complete model in Fig. 5. In comparison, during the entire duration of the isolated CP El Niño the SSTs remain warm over the tropical Pacific. As a result, the cloud radiative feedback dominantly warms the central Pacific, including during the buildup and aftermath of the event at year -1 and +1 , respectively. The role of this seasonal feedback is therefore significantly different for the EP or CP El Niño in the model.

\section{d. Additional seasonal feedbacks}

A great diversity of processes may be responsible for the seasonal synchronization of ENSO in nature beyond the cloud radiative feedback analyzed in previous sections. For completeness we analyze here the ENSO model's sensitivity to other possible seasonal feedbacks as suggested from the literature. For this, we consider here additional numerical experiments where instead of the cloud radiative feedback we vary seasonally either a wind stress (WS), wind burst (WB), or thermocline (TH) feedback as well as a bulk SST feedback (BLK). Model modifications for each experiment are provided in Table A3. Although a realistic ENSO model should include a balanced prescribed background state with all potential seasonal feedbacks, important lessons can be learned here using the present artificial separation between feedbacks.

The histograms in Figs. 7a,d and 8a,d show that a qualitative ENSO seasonal synchronization is achieved 
(a) WS: Histogram (events/yr)

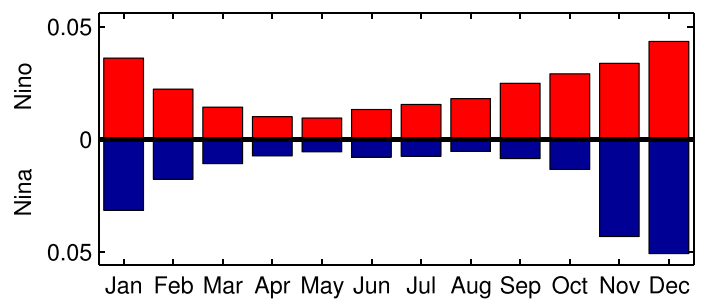

(b) $\gamma_{s}(\tau)$

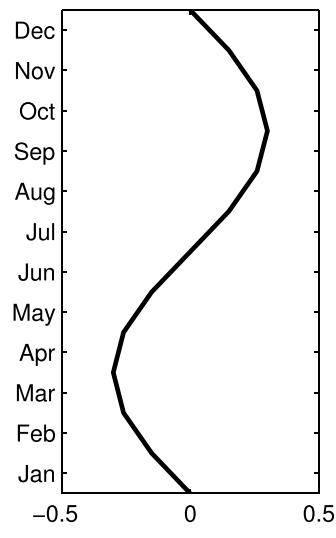

(c) $\gamma_{s} u$

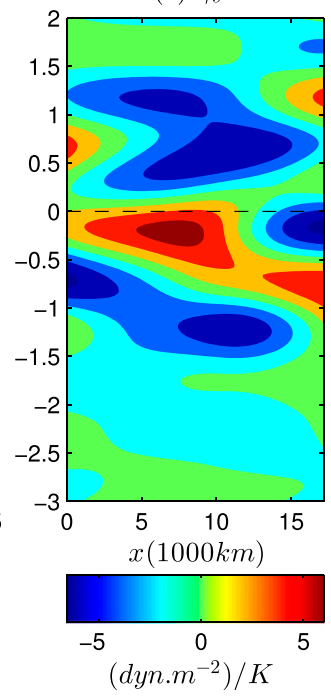

(d) WB: Histogram (events/yr)

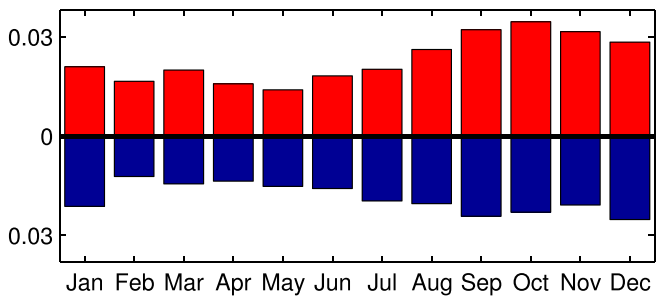

(e) $\gamma_{s p}(\tau)$
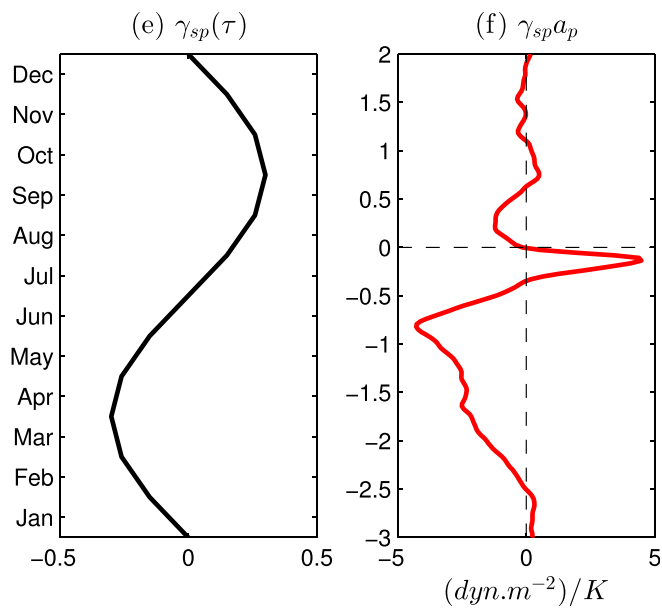

FIG. 7. Experiment with seasonal WS: (a) histogram of El Niño (red) and La Niña (blue) events peaks (events per year), (b) seasonal perturbation parameter $\gamma_{s}(\tau)$ as a function of month of year, and (c) lagged regression of wind stress forcing $\gamma_{s} u$ on Niño-3.4 SST $\left(\operatorname{dyn~} \mathrm{m}^{-2} \mathrm{~K}^{-1} ; 1 \mathrm{dyn}=10^{-5} \mathrm{~N}\right)$, as a function of zonal position $(1000 \mathrm{~km})$ and lead time (yr). Experiment with seasonal WB: (d) histogram, (e) seasonal perturbation parameter $\gamma_{\mathrm{sp}}(\tau)$, and (f) lagged regression of wind stress forcing $\gamma_{\mathrm{sp}} a_{p}$ on Niño-3.4. SST (dyn $\mathrm{m}^{-2} \mathrm{~K}^{-1}$ ).

for each of the additional experiments $\mathrm{WS}, \mathrm{WB}, \mathrm{TH}$, and BLK. Other important statistical and dynamical features of the model as described in previous sections are also conserved overall, although we do not document these here for brevity. Despite this apparent similarity between experiments, there are several important strengths and weaknesses in the formulation of each seasonal feedback and their contribution to the life cycle of ENSO events, as discussed below. Note that the cloud radiative feedback in comparison has the advantage of being self-consistently derived with clear observational analogues and model solutions robust to the parameter perturbation.

In experiment WS (Figs. 7a-c), we vary seasonally the wind stress feedback (or Bjerknes feedback; Zebiak and Cane 1987; Jin et al. 2006), that is, the strength of the remote wind stress response to SSTs, as a result for example of the seasonal motion of the intertropical convergence zone (ITCZ) (Tziperman et al. 1997) or the southward shift of zonal winds in spring (Lengaigne et al. 2006; Stuecker et al. 2013). For this we add a wind stress forcing term $\gamma_{s} u$ in the model, with seasonal parameter $\gamma_{s}$ maximal in fall and minimal in spring (Fig. 7b) and varying within $\pm 5 \%$ of the value of $\gamma$. Note that increased variations $( \pm 20 \%)$ lead to an unrealistic ENSO variability and lack of robustness (not shown). The lagged regressions in Fig. 7c highlight the contribution of the wind stress forcing term $\gamma_{s} u$ to the chronology of ENSO events in the model (in fashion similar to the cloud radiative feedback heating contribution in Fig. 5f). The contribution of $\gamma_{s} u$ in the central Pacific consistently favors the growth and demise of ENSO events by reinforcing the zonal winds from around -0.5 to $0 \mathrm{yr}$ prior to the peak and decreasing them from around +0.5 to $+1 \mathrm{yr}$ in the aftermath. However, because of the remote nature of the present feedback there are other significant contributions in the far western or eastern Pacific as well as during the prior or following years. For example, there are positive contributions in the far western $\mathrm{Pa}$ cific at $+0.7 \mathrm{yr}$ and in the far eastern Pacific at $+1.2 \mathrm{yr}$, and so on. As compared to the cloud radiative 
(a) TH: Histogram (events/yr)

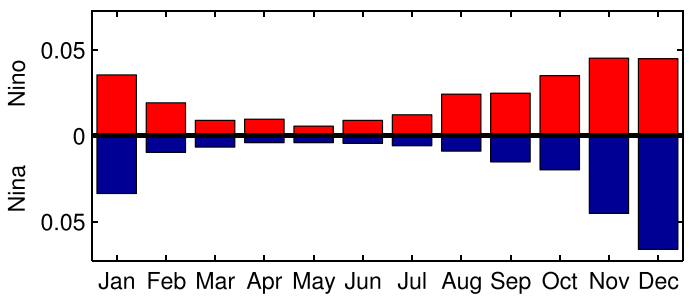

(b) $\eta_{s}(x, \tau)$

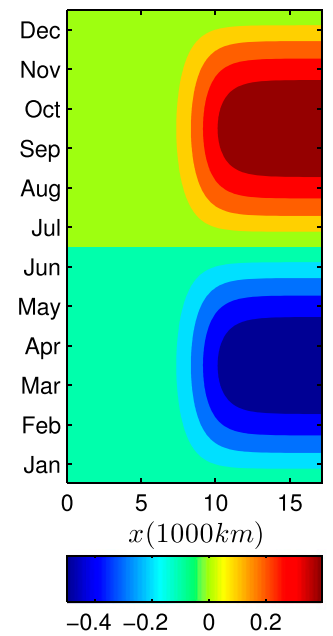

(c) $\eta_{s} H$

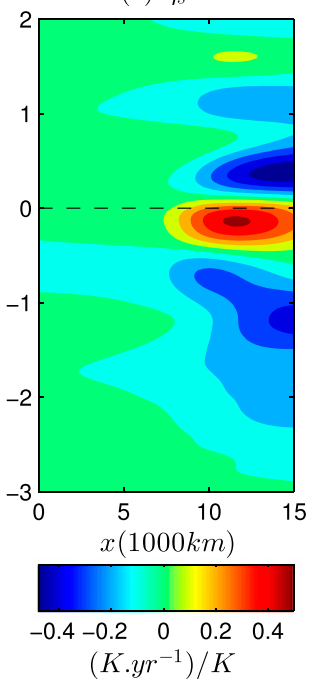

(d) BLK: Histogram (events/yr)

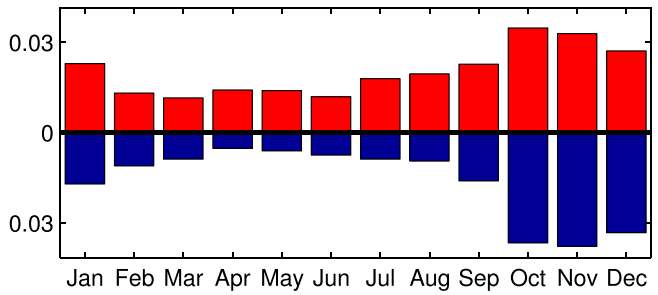

(e) $\alpha_{B}(\tau)$

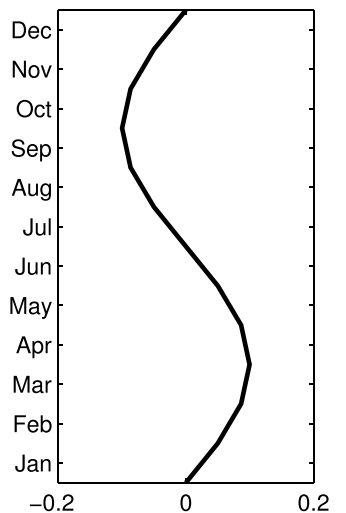

(f) $\alpha^{B} M\left(T_{30}-\bar{T}-T\right)$

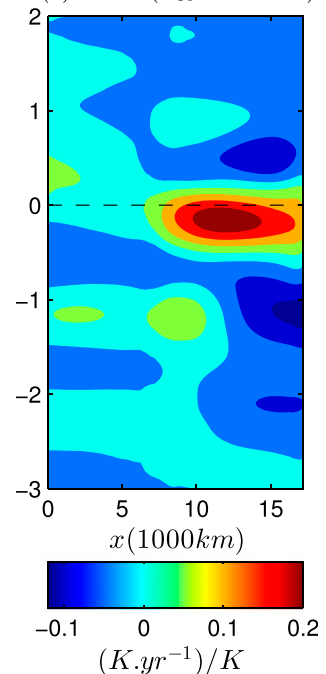

FIG. 8. Experiment with seasonal TH: (a) histogram of El Niño (red) and La Niña (blue) events peaks (events per year), (b) seasonal perturbation parameter $\eta_{s}(x, \tau)$ as a function of zonal position $(1000 \mathrm{~km})$ and month of year, and (c) lagged regression of heating $\eta_{s} H$ on Niño-3.4 SST $\left(\mathrm{K} \mathrm{yr}^{-1} \mathrm{~K}^{-1}\right)$, as a function of zonal position $(1000 \mathrm{~km})$ and lead time (yr). the Experiment with seasonal BLK: (d) histogram, (e) seasonal perturbation parameter $\alpha^{B}(\tau)$, and (f) lagged regression of heating $\alpha^{B}(\tau) M\left[T_{30}-\bar{T}(x)-T\right]$ on Niño-3.4. SST $\left(\mathrm{K} \mathrm{yr}^{-1} \mathrm{~K}^{-1}\right)$.

feedback those remote contributions have no clear analogues in the observational record and it is unclear to which extent they affect the chronology of ENSO events in the model; note, however, that those contributions could be related to combination tones from the interaction of ENSO and the seasonal cycle (Stuecker et al. 2013).

In experiment WB (Figs. 7d-f), we vary in a similar fashion the wind burst feedback (i.e., the wind bursts response to SSTs; Hendon et al. 2007; Seiki and Takayabu 2007) by adding a wind burst forcing term $\gamma_{\mathrm{sp}} a_{p}$ with seasonal parameter $\gamma_{\mathrm{sp}}$ (Fig. 7e). For instance, if the strength of the remote wind stress response to SSTs shows seasonal variations then so should the wind bursts response, under similar arguments. As shown in the lagged regressions (Fig. 7f) the term $\gamma_{\mathrm{sp}} a_{p}$ reinforces the easterly wind bursts contribution during the buildup phase of El Niño events (from -1 to $-2 \mathrm{yr}$ ) as well as the westerly wind bursts contribution during the trigger phase (from -0.5 to $0 \mathrm{yr}$ ), although there are no clear observational analogues for this. In addition, this seasonal feedback unrealistically tends to exaggerate the peaking of ENSO events in September-October as well as their spread throughout the year (Fig. 7d). In addition to the wind bursts response to SST, another important feature of wind bursts in nature is their increased occurrence and frequency in boreal winter (cf. Fig. 1; Lopez and Kirtman 2014). This could be accounted for in the model by varying seasonally the source of wind bursts $\sigma_{p}$. Note, however, that varying $\sigma_{p}$ or the present parameter $\gamma_{\mathrm{ps}}$ is dynamically identical as can be seen by rescaling Eq. (6). If we were to account instead for the increased frequency of wind bursts in boreal winter (through an increase of $\sigma_{p}$ or equivalently of $\gamma_{\mathrm{ps}}$ ) the peaking of ENSO events in Fig. 7d would be more realistically shifted toward November-December.

In experiment TH (Figs. 8a-c), we vary seasonally the thermocline feedback (Hirst 1986; Tziperman et al. 1997; Galanti et al. 2002) by adding a heating term $\eta_{s} H$ in the model's SST budget with a seasonal parameter $\eta_{s}$ $(x, \tau)$ that is maximal in fall and minimal in spring in the eastern Pacific (Fig. 8b). To achieve seasonal 
synchronization, we vary $\eta_{s}$ within $+25 \%$ of the value of $\eta$ (Fig. 2a); however, in nature it is unclear whether those variations are as marked. For instance, while interannual variations of thermocline depth and upwelling are pronounced their climatological variations are weaker in comparison (e.g., Figs. 1d and 1i). Despite this, the heating contribution of $\eta_{s} H$ is similar to the one of the cloud radiative feedback during the lifecycle of ENSO events (Fig. 8c).

Finally, in experiment BLK (Figs. 8d-f), we propose as a complementary result a heuristic bulk SST feedback motivated by the observed SST climatology in Fig. 1c. Although the bulk SST feedback lacks simplicity compared to the cloud radiative feedback for practical implementations, it is dynamically similar and illustrates how more details of the state dependency on SST may be accounted for (Guilyardi et al. 2009; Lloyd et al. 2012) as a motivation for future work. For instance, as shown in Fig. 1c, whereas SSTs in the eastern Pacific show marked seasonal changes, the warm SSTs in the western Pacific show no seasonal sensitivity. The simplest modification of the SST budget that embodies this idea is

$$
\begin{aligned}
\partial_{\tau} T+\mu \partial_{x}(U T)= & -c_{1} \zeta E_{q}+c_{1} \eta H \\
& +c_{1} \alpha^{B}(\tau) M\left[T_{30}-\bar{T}(x)-T\right],
\end{aligned}
$$

where $\alpha^{B}(\tau)$ accounts for seasonal variations in a fashion that is similar to the cloud radiative feedback (Fig. 8e), $M(x)=x$ if $x \geq 0$ and is zero otherwise, $T_{30}=$ $30^{\circ} \mathrm{C}$ is the threshold total temperature above which seasonal sensitivity vanishes as in the western Pacific, and $\bar{T}(x)$ is the background mean temperature (around $30^{\circ} \mathrm{C}$ in the western Pacific and $20^{\circ} \mathrm{C}$ in the eastern Pacific). As shown in Fig. 8f, the bulk SST feedback has a similar contribution as the cloud radiative feedback during the life cycle of El Niño events. However, the present feedback unrealistically tends to exaggerate the peaking of ENSO events in October-November (Fig. 8d).

\section{Discussion}

In the present article, we have analyzed the seasonal synchronization of a simple stochastic dynamical model that captures the ENSO diversity and intermittency in nature. Given the realism of such a model and its potential implications for studying ENSO's mechanisms (Thual et al. 2016; Chen and Majda 2016a,b), its capacity to reproduce major aspects of the ENSO seasonal synchronization in nature
TABLE A1. Model variables with definitions and values with units.

\begin{tabular}{llc}
\hline \hline & \multicolumn{1}{c}{ Variable } & Value \\
\hline$x$ & Zonal axis & $15000 \mathrm{~km}$ \\
$y$ & Meridional axis atmosphere & $1500 \mathrm{~km}$ \\
$Y$ & Meridional axis ocean & $330 \mathrm{~km}$ \\
$\tau$ & Time axis (interannual) & 33 days \\
$u$ & Zonal wind speed anomalies & $5 \mathrm{~m} \mathrm{~s}^{-1}$ \\
$v$ & Meridional wind speed anomalies & $0.5 \mathrm{~m} \mathrm{~s}^{-1}$ \\
$\theta$ & Potential temperature anomalies & $1.5 \mathrm{~K}$ \\
$a$ & Envelope of synoptic convective activity & 1 \\
$H a$ & Convective heating/drying & $0.45 \mathrm{~K} \mathrm{day}^{-1}$ \\
$E_{q}$ & Latent heating anomalies & $0.45 \mathrm{~K} \mathrm{day}^{-1}$ \\
$T$ & Sea surface temperature anomalies & $1.5 \mathrm{~K}^{-1}$ \\
$U$ & Zonal current speed anomalies & $0.25 \mathrm{~m} \mathrm{~s}^{-1}$ \\
$V$ & Meridional current speed anomalies & $0.56 \mathrm{~cm} \mathrm{~s}^{-1}$ \\
$H$ & Thermocline depth anomalies & $20.8 \mathrm{~m} \mathrm{~m}^{-2}$ \\
$\tau_{x}$ & Zonal wind stress anomalies & $0.00879 \mathrm{~N} \mathrm{~m}^{-2}$ \\
\hline
\end{tabular}

is an important requirement. This goal is achieved here thanks to the addition of a cloud radiative feedback that accounts for seasonal variations in cloud cover and convective activity in the eastern Pacific in simple fashion. Generally speaking, the present work suggests that the seasonality of the equatorial Pacific should not be ignored even in simplified ENSO models.

The seasonal cloud radiative feedback here accounts for the weak convective response to SSTs in boreal fall that favors the development of El Niño events in the eastern Pacific as well as the increased convective activity and cloud cover in the following spring that contributes to the shutdown of those events by blocking incoming shortwave solar radiations, in overall agreement with the literature (Lloyd et al. 2012; Dommenget and Yu 2016). Importantly, the cloud radiative feedback is derived here naturally from the model's atmosphere with no ad hoc assumptions: in particular, a simple collective representation of convective processes is considered in the model's atmosphere that has been successful in other settings to realistically capture the most salient features of the $\mathrm{MJO}$ and intraseasonal variability in the tropics (Majda and Stechmann 2009, 2011; Stechmann and Majda 2015). It should be noted, however, that the present parameterization omits the detailed radiative or thermodynamic effects that different types of clouds (e.g., low-level or upper-level clouds) can have on the SST budget (Frenkel et al. 2015). This needs to be addressed in future studies. Nevertheless, the present work and formulation of the cloud radiative feedback may have implications for understanding the seasonal synchronization of ENSO in CGCMs. The intimate dependency of ENSO on the 
TABLE A2. Model parameter definitions and nondimensional values.

\begin{tabular}{lcc}
\hline \hline & Parameter & Value \\
\hline$c_{1}$ & Ocean phase speed & 0.5 \\
$L_{A}$ & Equatorial belt length & $8 / 3$ \\
$L_{O}$ & Equatorial Pacific length & 1.2 \\
$\bar{H}$ & Convective heating rate & 22 \\
$\bar{Q}$ & Vertical moisture gradient & 0.9 \\
$\gamma$ & Wind stress coefficient & 6.53 \\
$\alpha_{q}$ & Latent heating factor & 0.2 \\
$\zeta$ & Latent heating capacity & 8.7 \\
$d_{p}$ & Wind burst damping & 5.1 \\
& Wind burst structure at equator: $s_{p}(x)=\exp \left[-45\left(x-L_{O} / 4\right)^{2}\right]$ \\
& Thermocline feedback at equator: $\eta(x)=1.5+0.5 \tanh \left(7.5 x-L_{O} / 2\right)$ \\
\hline
\end{tabular}

seasonal cycle presents a major challenge for those models as they still show major deficiencies in simulating the ENSO amplitude and frequency, spatial structure, and seasonal synchronization due to systematic biases in the mean climate and seasonal cycle of the tropical Pacific (Guilyardi 2006; Lloyd et al. 2012; Bellenger et al. 2014). Among those biases, the cloud radiative feedback in the eastern Pacific remains a major source of uncertainty (Bony and Dufresne 2005; Lloyd et al. 2012; Guilyardi et al. 2009; Bellenger et al. 2014).

While the cloud radiative feedback certainly plays a key role for the seasonal synchronization of ENSO, other possible seasonal feedbacks may also be important and their incorporation in the ENSO model has also been documented for completeness. For instance, the present model may also capture qualtitatively the ENSO seasonal synchronization due to seasonal changes in wind stress (Zebiak and Cane 1987; Jin et al. 2006; Tziperman et al. 1997), wind bursts (Hendon et al. 2007; Seiki and Takayabu 2007), or thermocline feedback (Hirst 1986; Tziperman et al. 1997; Galanti et al. 2002) as well as with a simple heuristic bulk SST feedback. There are several important strengths and weaknesses in the formulation of each seasonal feedback and its contribution to the life cycle of ENSO events: this sometimes includes ad hoc formulations, lack of robustness, or observational analogues. The cloud radiative feedback in comparison has the advantage to be self-consistently derived with clear observational analogues, as discussed above. For future work a more complete ENSO model with a balanced prescribed background state should be considered in order to compare the interplay between each of those seasonal feedbacks. In particular, as documented in the present article, seasonal feedbacks play different roles at different stages of the life cycle of El Niño events (e.g., during their buildup, trigger, or shutdown), which should be studied in more detail. Finally, in nature a large diversity of processes are involved in the ENSO dynamics that are not considered here. For example, several potentially important seasonal feedbacks have been disregarded such as the seasonal cycle of currents in the eastern Pacific (Mitchell and Wallace 1992). In addition, a more detailed representation of the intraseasonal wind burst activity should be included in the model (Majda and Stechmann 2009, 2011; Thual et al. 2014).

Acknowledgments. The research of A.J.M. is partially supported by the Office of Naval Research Grant ONR MURI N00014-16-1-2161. S.T. and N.C. are supported as postdoctoral fellows through A.J. M.'s ONR MURI Grant. The authors thank S.N. Stechmann for useful discussions on the cloud radiative feedback parameterization.

TABLE A3. Model parameterization and modifications for all seasonal feedbacks.

\begin{tabular}{lc}
\hline \hline \multicolumn{1}{c}{ Seasonal feedback } & Model parameterization \\
\hline Cloud radiative feedback & $\partial_{\tau} T+\mu \partial_{x}(U T)=-c_{1} \zeta E_{q}+c_{1} \eta H-c_{1} \alpha T, \alpha(x, \tau)=0.2 \sin (\omega \tau) s_{m}(x)$ \\
& $\omega=2 \pi / 1 \mathrm{yr}^{-1}:$ annual cycle period, $s_{m}(x)=\left\{\tanh \left[7.5\left(x-L_{O} / 2\right)\right]+1\right\} / 2$ \\
Wind stress feedback & $\partial_{\tau} T+\mu \partial_{x}(U T)=-c_{1} \zeta E_{q}+c_{1} \eta H, \tau_{x}=\gamma\left(u+u_{p}\right)+\gamma_{s} u, \gamma_{s}=-0.3 \sin (\omega \tau)$. \\
Wind burst feedback & $\partial_{\tau} T+\mu \partial_{x}(U T)=-c_{1} \zeta E_{q}+c_{1} \eta H, \tau_{x}=\gamma\left(u+u_{p}\right)+\gamma_{\mathrm{sp}} u_{p} \gamma_{\mathrm{sp}}=-0.3 \sin (\omega \tau)$. \\
Thermocline feedback & $\partial_{\tau} T+\mu \partial_{x}(U T)=-c_{1} \zeta E_{q}+c_{1} \eta H, \eta_{s}=-0.5 \sin (\omega \tau) s_{m}(x)$. \\
Bulk SST feedback & $\alpha^{B}(T)=0.1 \sin (\omega \tau), T_{30}=20\left(30^{\circ} \mathrm{C}\right.$ in dimensional units $), \bar{T}(x)=T_{30}-6.66 s_{m}(x)$. \\
\hline
\end{tabular}




\section{APPENDIX A}

\section{Model Tables}

This appendix provides additional details on the coupled ENSO model. Table A1 provides all variables, with definition and units. Table A2 provides all parameter definitions and nondimensional values. Table A3 details the model parameterization for the cloud radiative feedback and modifications for the experiments with additional seasonal feedbacks from section $3 \mathrm{~d}$. Note that in each experiment a single parameter is varied seasonally, where the variations are sinusoidal with a zero-background time mean.

\section{APPENDIX B}

\section{Asymptotic Expansion}

This appendix details the asymptotic expansion at interannual time scale for the skeleton model, from Eq. (10) to Eqs. (1) and (11) in the main body of the present article. First, we replace intraseasonal time $t$ with interannual time $\tau=\varepsilon t$ in Eq. (10), where $\varepsilon=0.1$ is the Froude number [see the supplemental information of Thual et al. (2016)]. This reads:

$$
\begin{aligned}
\varepsilon \partial_{\tau} u-y v-\partial_{x} \theta & =0, \\
y u-\partial_{y} \theta & =0, \\
\varepsilon \partial_{\tau} \theta-\left(\partial_{x} u+\partial_{y} v\right) & =\bar{H} a-s^{\theta}, \\
\varepsilon \partial_{\tau} q+\bar{Q}\left(\partial_{x} u+\partial_{y} v\right) & =-\bar{H} a+s^{q}+E_{q}, \\
\varepsilon \partial_{\tau} a & =\Gamma q a .
\end{aligned}
$$

Second, we consider an asymptotic expansion of the above system in powers of $\varepsilon$, with the generic form $\mathbb{U}=\sum_{n=0}^{N} \mathbb{U}_{n} \varepsilon^{n}+o\left(\varepsilon^{n}\right)$, where $\mathbb{U}=\left\{u, v, \theta, a, E_{q}\right\}$. Retaining the first-order $n=0$ of the asymptotic expansion, the system reads as follows:

$$
\begin{aligned}
-y v-\partial_{x} \theta & =0, \\
y u-\partial_{y} \theta & =0, \\
-\left(\partial_{x} u+\partial_{y} v\right) & =\bar{H} a-s^{\theta}, \\
\bar{Q}\left(\partial_{x} u+\partial_{y} v\right) & =-\bar{H} a+s^{q}+E_{q}, \\
q & =0,
\end{aligned}
$$

where we recall that $a \geq 0$ in the above system. In particular, convective activity $a$ can be expressed as

$$
\bar{H} a=\left(s^{q}-\bar{Q}^{\theta}+E_{q}\right) /(1-\bar{Q}),
$$

where we further separate a background component $\bar{H} \bar{a}=\left(s^{q}-\bar{Q} s^{\theta}\right) /(1-\bar{Q})$ that results from the adjustment to the constant external sources $s^{\theta}$ and $s^{q}$. We obtain the interannual atmosphere model used in the ENSO model as described in Eqs. (1) and (11).

\section{REFERENCES}

An, S.-I., and F.-F. Jin, 2001: Collective role of thermocline and zonal advective feedbacks in the ENSO mode. J. Climate, 14, 3421-3432, doi:10.1175/1520-0442(2001)014<3421: CROTAZ $>2.0 . \mathrm{CO} ; 2$.

Behringer, D., M. Ji, and A. Leetmaa, 1998: An improved coupled model for ENSO prediction and implications for ocean initialization. Part I: The ocean data assimilation system. Mon. Wea. Rev., 126, 1013-1021, doi:10.1175/1520-0493(1998)126<1013: AICMFE $>2.0 . \mathrm{CO} ; 2$.

Bellenger, H., E. Guilyardi, J. Leloup, M. Lengaigne, and J. Vialard, 2014: ENSO representation in climate models: From CMIP3 to CMIP5. Climate Dyn., 42, 1999-2018, doi:10.1007/s00382-013-1783-z.

Bony, S., and J.-L. Dufresne, 2005: Marine boundary layer clouds at the heart of tropical cloud feedback uncertainties in climate models. Geophys. Res. Lett., 32, L20806, doi:10.1029/2005GL023851.

Chen, D., and Coauthors, 2015: Strong influence of westerly wind bursts on El Niño diversity. Nat. Geosci., 8, 339-345, doi:10.1038/ngeo2399.

Chen, N., and A. Majda, 2015: Predicting the real-time multivariate Madden-Julian oscillation index through a low-order nonlinear stochastic model. Mon. Wea. Rev., 143, 2148-2169, doi:10.1175/MWR-D-14-00378.1.

— features of the central Pacific El Niño. Proc. Natl. Acad. Sci. USA, 113, 11 732-11 737, doi:10.1073/pnas.1614533113.

— , and,$- 2016 \mathrm{~b}$ : Simple stochastic dynamical models capturing the statistical diversity of El Niño Southern Oscillation. Proc. Natl. Acad. Sci. USA, 113, 11732-11737, doi:10.1073/ pnas.1614533113.

— - — and S. Thual, 2017: Observations and mechanisms of a simple stochastic dynamical model capturing El Niño diversity. J. Climate, in press.

Clarke, A. J., 2008: An Introduction to the Dynamics of El Niño and the Southern Oscillation. Academic Press, 324 pp.

— equatorial Pacific phase-locking El Niño to the seasonal cycle. Geophys. Res. Lett., 27, 771-774, doi:10.1029/1999GL010506.

Dewitte, B., S.-W. Yeh, and S. Thual, 2013: Reinterpreting the thermocline feedback in the western-central equatorial $\mathrm{Pa}$ cific and its relationship with the ENSO modulation. Climate Dyn., 41, 819-830, doi:10.1007/s00382-012-1504-z.

Dommenget, D., and Y. Yu, 2016: The seasonally changing cloud feedbacks contribution to the ENSO seasonal phase-locking. Climate Dyn., 47, 3661-3672, doi:10.1007/s00382-016-3034-6.

_, S. Haase, T. Bayr, and C. Frauen, 2014: Analysis of the slab ocean El Niño atmospheric feedbacks in observed and simulated ENSO dynamics. Climate Dyn., 42, 3187-3205, doi:10.1007/s00382-014-2057-0.

Fedorov, A., 2002: The response of the coupled tropical oceanatmosphere to westerly wind bursts. Quart. J. Roy. Meteor. Soc., 128, 1-23, doi:10.1002/qj.200212857901. 
Frenkel, Y., A. Majda, and S. Stechmann, 2015: Cloud-radiation feedback and atmosphere-ocean coupling in a stochastic multicloud model. Dyn. Atmos. Oceans, 71, 35-55, doi:10.1016/j.dynatmoce.2015.05.003.

Galanti, E., E. Tziperman, M. Harrison, A. Rosati, R. Giering, and Z. Sirkes, 2002: The equatorial thermocline outcropping-A seasonal control on the tropical Pacific Ocean-atmosphere instability strength. J. Climate, 15, 2721-2739, doi:10.1175/ 1520-0442(2002)015<2721:TETOAS > 2.0.CO;2.

Gardiner, C. W., 1994: Handbook of Stochastic Methods for Physics, Chemistry, and the Natural Sciences. 2nd ed. Springer, 442 pp.

Gill, A., 1980: Some simple solutions for heat-induced tropical circulation. Quart. J. Roy. Meteor. Soc., 106, 447-462, doi:10.1002/qj.49710644905.

Guilyardi, E., 2006: El Niño-mean state-seasonal cycle interactions in a multi-model ensemble. Climate Dyn., 26, 329348, doi:10.1007/s00382-005-0084-6.

- , P. Braconnot, F.-F. Jin, S. T. Kim, M. Kolasinski, T. Li, and I. Musat, 2009: Atmosphere feedbacks during ENSO in a coupled GCM with a modified atmospheric convection scheme. J. Climate, 22, 5698-5712, doi:10.1175/2009JCLI2815.1.

Hendon, H., M. Wheeler, and C. Zhang, 2007: Seasonal dependence of the MJO-ENSO relationship. J. Climate, 20, 531543, doi:10.1175/JCLI4003.1.

Hirst, A., 1986: Unstable and damped equatorial modes in simple coupled ocean-atmosphere models. J. Atmos. Sci., 43, 606-632, doi:10.1175/1520-0469(1986)043\%3C0606: UADEMI\%3E2.0.CO;2.

Jin, F.-F., J. Neelin, and M. Ghil, 1994: El Niño on the devil's staircase: Annual subharmonic steps to chaos. Science, 264, 70-72, doi:10.1126/science.264.5155.70.

_ - S. T. Kim, and L. Bejarano, 2006: A coupled-stability index for ENSO. Geophys. Res. Lett., 33, L23708, doi:10.1029/ 2006 GL027221.

_ , L. Lin, A. Timmermann, and J. Zhao, 2007: Ensemble-mean dynamics of the ENSO recharge oscillator under statedependent stochastic forcing. Geophys. Res. Lett., 34, L03807, doi:10.1029/2006GL027372.

Kalnay, E., and Coauthors, 1996: The NCEP/NCAR 40-Year Reanalysis Project. Bull. Amer. Meteor. Soc., 77, 437-471, doi:10.1175/1520-0477(1996)077<0437:TNYRP>2.0.CO;2.

Kleeman, R., 2008: Stochastic theories for the irregularity of ENSO. Philos. Trans. Roy. Soc. London, 366A, 2509-2524, doi:10.1098/rsta.2008.0048.

Lawler, G. F., 2006: Introduction to Stochastic Processes. Chapman and Hall, $192 \mathrm{pp}$

Lengaigne, M., J.-P. Boulanger, C. Menkes, and H. Spencer, 2006: Influence of the seasonal cycle on the termination of El Niño events in a coupled general circulation model. J. Climate, $\mathbf{1 9}$, 1850-1868, doi:10.1175/JCLI3706.1.

Levine, A., and M. McPhaden, 2015: The annual cycle in ENSO growth rate as a cause of the spring predictability barrier. Geophys. Res. Lett., 42, 5034-5041, doi:10.1002/ 2015 GL064309.

Liebmann, B., and C. Smith, 1996: Description of a complete (interpolated) outgoing longwave radiation dataset. Bull. Amer. Meteor. Soc., 77, 437-471, https://www.esrl.noaa.gov/psd/data/ gridded/OLRinterpolated.pdf.

Lloyd, J., E. Guilyardi, and H. Weller, 2012: The role of atmosphere feedbacks during ENSO in the CMIP3 models. Part III: The shortwave flux feedback. J. Climate, 25, 4275-4293, doi:10.1175/JCLI-D-11-00178.1.
Lopez, H., and B. Kirtman, 2014: WWBs, ENSO predictability, the spring barrier and extreme events. J. Geophys. Res. Atmos., 119, 10 114-10 138, doi:10.1002/2014JD021908.

Majda, A. J., 2003: Introduction to PDEs and Waves for the Atmosphere and Ocean. Courant Lecture Notes in Mathematics, Vol. 9, American Mathematical Society, 234 pp.

- - , and R. Klein, 2003: Systematic multiscale models for the tropics. J. Atmos. Sci., 60, 393-408, doi:10.1175/ 1520-0469(2003)060<0393:SMMFTT $>2.0$. CO;2.

- and S. N. Stechmann, 2009: The skeleton of tropical intraseasonal oscillations. Proc. Natl. Acad. Sci. USA, 106, 84178422, doi:10.1073/pnas.0903367106.

, and _ 2011: Nonlinear dynamics and regional variations in the MJO skeleton. J. Atmos. Sci., 68, 3053-3071, doi:10.1175/JAS-D-11-053.1.

, and J. Harlim, 2012: Filtering Complex Turbulent Systems. Cambridge University Press, $368 \mathrm{pp}$.

Mitchell, T., and J. Wallace, 1992: The annual cycle in equatorial convection and sea surface temperature. J. Climate, 5, 1140-1156, doi:10.1175/1520-0442(1992)005<1140: TACIEC $>2.0 . \mathrm{CO} ; 2$.

Moore, A. M., and R. Kleeman, 1999: Stochastic forcing of ENSO by the intraseasonal oscillation. J. Climate, 12, 1199-1220, doi:10.1175/1520-0442(1999)012<1199:SFOEBT >2.0.CO;2.

Neelin, J. D., D. S. Battisti, A. C. Hirst, F.-F. Jin, Y. Wakata, T. Yamagata, and S. E. Zebiak, 1998: ENSO theory. J. Geophys. Res., 103, 14 261-14 290, doi:10.1029/97JC03424.

, F.-F. Jin, and H.-H. Syu, 2000: Variations in ENSO phase locking. J. Climate, 13, 2570-2590, doi:10.1175/1520-0442(2000)013<2570: VIEPL $>2.0 . \mathrm{CO} ; 2$

Ogrosky, H. R., and S. N. Stechmann, 2015: The MJO skeleton model with observation-based background state and forcing. Quart. J. Roy. Meteor. Soc., 141, 2654-2669, doi:10.1002/ qj.2552.

Philander, S., and A. Fedorov, 2003: Is El Niño sporadic or cyclic? Annu. Rev. Earth Planet. Sci., 31, 579-594, doi:10.1146/ annurev.earth.31.100901.141255.

Puy, M., J. Vialard, M. Lengaigne, and E. Guilyardi, 2016: Modulation of equatorial Pacific westerly/easterly wind events by the Madden-Julian oscillation and convectively-coupled Rossby waves. Climate Dyn., 46, 2155-2178, doi:10.1007/ s00382-015-2695-x.

Reynolds, R., T. Smith, C. Liu, D. B. Chelton, K. S. Casey, and M. G. Schlax, 2007: Daily high-resolution-blended analyses for sea surface temperature. J. Climate, 20, 5473-5496, doi:10.1175/2007JCLI1824.1.

Seiki, A., and Y. Takayabu, 2007: Westerly wind bursts and their relationship with intraseasonal variations and ENSO. Part I: Statistics. Mon. Wea. Rev., 135, 3325-3345, doi:10.1175/ MWR3477.1.

Stachnik, J. P., D. E. Waliser, and A. Majda, 2015: Precursor environmental conditions associated with the termination of Madden-Julian oscillation events. J. Atmos. Sci., 72, 19081931, doi:10.1175/JAS-D-14-0254.1.

Stechmann, S. N., and H. Ogrosky, 2014: The Walker circulation, diabatic heating, and outgoing longwave radiation. Geophys. Res. Lett., 41, 9097-9105, doi:10.1002/2014GL062257.

, and A. J. Majda, 2015: Identifying the skeleton of the Madden-Julian oscillation in observational data. Mon. Wea. Rev., 143, 395-416, doi:10.1175/MWR-D-14-00169.1.

Stein, K., A. Timmerman, N. Schneider, F.-F. Jin, and M. Stuecker, 2014: ENSO seasonal synchronization theory. J. Climate, 27, 5285-5310, doi:10.1175/JCLI-D-13-00525.1. 
Stuecker, M., A. Timmerman, F. Jin, S. McGregor, and H. Ren, 2013: A combination mode of the annual cycle and the El Niño/Southern Oscillation. Nat. Geosci., 6, 540-544, doi:10.1038/ngeo1826.

Thual, S., A. J. Majda, and S. N. Stechmann, 2014: A stochastic skeleton model for the MJO. J. Atmos. Sci., 71, 697-715, doi:10.1175/JAS-D-13-0186.1.

, - - N. Chen, and S. Stechmann, 2016: Simple stochastic model for El Niño with westerly wind bursts. Proc. Natl. Acad. Sci. USA, 113, $10245-10250$, doi:10.1073/ pnas. 1612002113.

Torrence, C., and P. Webster, 1998: The annual cycle of persistence in the El Niño/Southern Oscillation. Quart. J. Roy. Meteor. Soc., 124, 1985-2004, doi:10.1002/qj.49712455010.

Tziperman, E., L. Stone, M. Cane, and H. Jarosh, 1994: El Niño chaos: Overlapping of resonances between the seasonal cycle and the Pacific Ocean-atmosphere oscillator. Science, 264, 7274, doi:10.1126/science.264.5155.72.

, S. Zebiak, and M. Cane, 1997: Mechanisms of seasonalENSO interaction. J. Atmos. Sci., 54, 61-71, doi:10.1175/ 1520-0469(1997)054\%3C0061:MOSEI\%3E2.0.CO;2.

Waliser, D., B. Blanke, J. Neelin, and C. Gautier, 1994: Shortwave feedbacks and El Niño-Southern Oscillation: Forced ocean and coupled ocean-atmosphere experiments. J. Geophys. Res., 99, 25 109-25 125, doi:10.1029/94JC02297.

Wang, W., and M. McPhaden, 2001: Surface layer temperature balance in the equatorial Pacific during the 1997-98 El Niño and 1998-99 La Niña. J. Climate, 14, 3393-3407, doi:10.1175/ 1520-0442(2001)014<3393:SLTBIT >2.0.CO;2.

Zebiak, S., and M. Cane, 1987: A model El Niño-Southern Oscillation. Mon. Wea. Rev., 115, 2262-2278, doi:10.1175/ 1520-0493(1987)115<2262:AMENO>2.0.CO;2. 